\title{
Galaxy and Mass Assembly (GAMA): Accurate number densities and environments of massive ultra-compact galaxies at $0.02<z<0.3^{\star}$
}

F. Buitrago ${ }^{1,2}$, I. Ferreras ${ }^{3}$, L. S. Kelvin ${ }^{4}$, I. K. Baldry ${ }^{4}$, L. Davies ${ }^{5}$, J. Angthopo ${ }^{3}$, S. Khochfar ${ }^{6}$, A. M. Hopkins ${ }^{7}$, S. P. Driver ${ }^{5}$, S. Brough ${ }^{8}$, J. Sabater ${ }^{6}$, C. J. Conselice ${ }^{9}$, J. Liske ${ }^{10}$, B. W. Holwerda ${ }^{11}$, M. N. Bremer ${ }^{12}$, S. Phillipps ${ }^{12}$, Á. R. López-Sánchez ${ }^{7,13}$, and A. W. Graham ${ }^{14}$

1 Instituto de Astrofísica e Ciências do Espaço, Universidade de Lisboa, OAL, Tapada da Ajuda, 1349-018 Lisbon, Portugal e-mail: fbuitrago@oal.ul.pt

2 Departamento de Física, Faculdade de Ciências, Universidade de Lisboa, Edifício C8, Campo Grande, 1749-016 Lisbon, Portugal

3 Mullard Space Science Laboratory, University College London, Holmbury St Mary, Dorking, Surrey RH5 6NT, UK e-mail: i.ferreras@ucl.ac.uk

${ }^{4}$ Astrophysics Research Institute, Liverpool John Moores University, IC2, Liverpool Science Park, 146 Brownlow Hill, Liverpool L3 5RF, UK

5 ICRAR, School of Physics, University of Western Australia, 35 Stirling Highway, Crawley, WA 6009, Australia

6 Institute for Astronomy, University of Edinburgh, Royal Observatory, Edinburgh EH9 3HJ, UK

7 Australian Astronomical Observatory, 105 Delhi Rd, North Ryde, NSW 2113, Australia

8 School of Physics, University of New South Wales, NSW 2052, Australia

9 Centre for Astronomy and Particle Theory, School of Physics \& Astronomy, University of Nottingham, Nottingham NG7 2RD, UK

10 Hamburger Sternwarte, Universität Hamburg, Gojenbergsweg 112, 21029 Hamburg, Germany

11 Department of Physics and Astronomy, 102 Natural Science Building, University of Louisville, Louisville, KY 40292, USA

12 H. H. Wills Physics Laboratory, University of Bristol, Tyndall Avenue, Bristol BS8 1TL, UK

13 Department of Physics and Astronomy, Macquarie University, NSW 2109, Australia

14 Centre for Astrophysics and Supercomputing, Swinburne University of Technology, Victoria 3122, Australia

Received 6 July 2018 / Accepted 3 September 2018

\section{ABSTRACT}

Context. Massive ultra-compact galaxies (MUGs) are common at $z=2-3$, but very rare in the nearby Universe. Simulations predict that the few surviving MUGs should reside in galaxy clusters, whose large relative velocities prevent them from merging, thus maintaining their original properties (namely stellar populations, masses, sizes and dynamical state).

Aims. Our goal is to obtain a complete census of the MUG population at $0.02<z<0.3$, determining the number density, population properties and environment.

Methods. We have taken advantage of the high-completeness, large-area spectroscopic GAMA survey, complementing it with deeper imaging from the KiDS and VIKING surveys. We find a set of 22 bona-fide MUGs, defined as having high stellar mass $\left(>8 \times 10^{10} M_{\odot}\right)$ and compact size $\left(R_{\mathrm{e}}<2 \mathrm{kpc}\right)$. An additional set of seven lower-mass objects $\left(6 \times 10^{10}<M_{\star} / M_{\odot}<8 \times 10^{10}\right)$ are also potential candidates according to typical mass uncertainties.

Results. The comoving number density of MUGs at low redshift $(z<0.3)$ is constrained at $(1.0 \pm 0.4) \times 10^{-6} \mathrm{Mpc}^{-3}$, consistent with galaxy evolution models. However, we find a mixed distribution of old and young galaxies, with a quarter of the sample representing (old) relics. MUGs have a predominantly early or swollen disk morphology (Sérsic index $1<n<2.5$ ) with high stellar surface densities $\left(\left\langle\Sigma_{\mathrm{e}}\right\rangle \sim 10^{10} M_{\odot} \mathrm{Kpc}^{-2}\right)$. Interestingly, a large fraction feature close companions - at least in projection - suggesting that many (but not all) reside in the central regions of groups. Halo masses show these galaxies inhabit average-mass groups.

Conclusions. As MUGs are found to be almost equally distributed among environments of different masses, their relative fraction is higher in more massive overdensities, matching the expectations that some of these galaxies fell in these regions at early times. However, there must be another channel leading some of these galaxies to an abnormally low merger history because our sample shows a number of objects that do not inhabit particularly dense environments.

Key words. galaxies: evolution - galaxies: clusters: general - galaxies: structure - Galaxy: fundamental parameters galaxies: elliptical and lenticular, $\mathrm{cD}$ - galaxies: stellar content

\footnotetext{
* Based on observations made with ESO Telescopes at the La Silla Paranal Observatory under programmes ID 179.A-2004 and ID 177.A-3016.
} 


\section{Introduction}

Massive galaxies - defined as having stellar masses exceeding the characteristic mass of the galaxy mass function $\left(M_{\star}\right)$, or roughly those with $\gtrsim 10^{11} M_{\odot}$ (see e.g. Baldry et al. 2012 ; Kelvin et al. 2014) - are privileged testbeds for galaxy evolution theories, as they generally underwent dramatic changes in their sizes, morphologies and star formation rates at an accelerated pace in comparison with lower mass objects across cosmic time (e.g. Daddi et al. 2005; Trujillo et al. 2006, 2007, 2011; Buitrago et al. 2008, 2013, 2014; van Dokkum et al. 2010; Conselice et al. 2011; Bruce et al. 2012; Ferreras et al. 2012; McLure et al. 2013; Driver et al. 2013; van der Wel et al. 2014 to name but a few; see also Khochfar \& Silk 2006; Naab et al. 2009; Oser et al. 2010; Johansson et al. 2012; Nipoti et al. 2012; Zolotov et al. 2015; Wellons et al. 2016; Genel et al. 2018; Lapi et al. 2018 from the theoretical point of view). Historically, a number of nearby massive galaxies were claimed to be rather compact as far back as Zwicky \& Kowal (1968), tentatively ascribing such a trend to cluster-related mechanisms (Strom \& Strom 1978).

At $z=2-3$, the majority of massive galaxies display very small sizes (effective radii $r_{\mathrm{e}} \leq 1-2 \mathrm{kpc}$ ) and thus they were branded "red nuggets" (Damjanov et al. 2009), with the term "red" adopted as some of them are already passive even at such early stages of cosmic evolution. It was also claimed that the nearby Universe was devoid of such ultracompact massive galaxies (e.g. Cimatti et al. 2008). Trujillo et al. (2009), later confirmed by Taylor et al. (2010) and Shih \& Stockton (2011), found a tiny (in terms of number density) population of such galaxies at $z<$ 0.2 . Nevertheless, the stellar populations of these newly found galaxies were rather young $(\sim 2 \mathrm{Gyr})$ and metal rich $\left(Z \sim Z_{\odot}\right)$, meaning that these objects were not survivors from the $z>2$ compact massive galaxy population (Ferré-Mateu et al. 2013). However, these results raised hopes of finding the local analogs of high- $z$ red nuggets, in other words nearby massive and small galaxies containing very old ( $>10 \mathrm{Gyr}$ ) stellar populations.

Poggianti et al. (2013; see also Valentinuzzi et al. 2010a,b) suggested that these surviving compact massive galaxies (the socalled relic galaxies) were to be found in galaxy clusters, where the high velocity dispersion in such overdensities would prevent further merging, as long as the galaxy entered the cluster early enough. This fact was later corroborated by simulations (Quilis \& Trujillo 2013; Stringer et al. 2015), although the actual number densities were under debate. The first positive detection of a relic galaxy was NGC 1277 (Trujillo et al. 2014), which is indeed part of a galaxy overdensity (the Perseus cluster). After this discovery, other papers followed, increasing the number of such extraordinary objects (Stockton et al. 2014; Ferré-Mateu et al. 2017; Y1ldırım et al. 2017). Nevertheless, the actual definition of a relic galaxy is somewhat arbitrary, because of the different compactness criteria, and disagreement regarding the determination of old stellar populations.

Our effort focuses on the selection of a bona-fide sample of nearby massive ultra-compact galaxies (MUGs), while detecting relics among them. Massive relic galaxies have exceptional properties. They display both large rotational velocity and velocity dispersion $\left(\gtrsim 300 \mathrm{~km} \mathrm{~s}^{-1}\right)$, show compelling evidence of a bottom-heavy IMF (Martín-Navarro et al 2015; Ferré-Mateu et al. 2017), host a uni-modal population of globular clusters (Beasley et al. 2018) and über-massive black holes (ÜMBHs; van den Bosch et al. 2012; Ferré-Mateu et al. 2015; Yıldırım et al. 2017). These ÜMBHs are potential outliers in the Magorrian et al. (1998) relation (see Fabian et al. 2013;
Yildırım et al. 2015; Scharwächter et al. 2016), for a contrasting view, see for instance Emsellem (2013) or Graham et al. (2016). It has been argued that this fact supports the view that the gas from these galaxies was stripped when falling into the high- $z$ overdensities, thus accounting for their passive nature and absence of size growth, but to date there is no conclusive observational determination about which environments these massive galaxies inhabit - there are hints in favour of galaxy overdensities in Damjanov et al. (2015) or Peralta de Arriba et al. (2016), while the opposite could be seen in Ferré-Mateu et al. (2017).

In a number of papers, authors have tried, and succeeded to identify these objects at lower redshift (Damjanov et al. 2014, 2015; Saulder et al. 2015; Tortora et al. 2016, 2018; Charbonnier et al. 2017). The difference between our work and theirs is that: 1) by resorting to the Galaxy and Mass Assembly survey (GAMA; Driver et al. 2011; Baldry et al. 2018), we are using the low-redshift survey with the highest spectroscopic completeness to date; 2) With the GAMA data, one could assess the kind of overdensities these objects inhabit - as we spectroscopically confirm the presence of neighbours (our completeness details could be found in Sects. 2.1 and 3.4); 3) by having spectra for all members of our target galaxy sample one could obtain both an accurate MUG number density as well as an estimate of their stellar ages (good enough to discern regular MUGs from potential galaxy relics). Moreover, our work introduces subtle differences about the characterization of the structural parameters, the stellar mass calculations and the inclusion or not of photometric redshifts (please also see the end Sect. 3.6 for a detailed enumeration of these caveats).

This paper is structured as follows: Sect. 2 shows the photometric and spectroscopic data and explains the galaxy selection criteria. Section 3 displays the number of tests applied to determine the structural and stellar population properties of the galaxies within our sample. Section 4 highlights our primary conclusions. Appendix A shows our simulations to determine the errors in our structural parameter determination. Appendix B contains tables of our inferred structural parameters. Our assumed cosmology is $\Omega_{\mathrm{m}}=0.3, \Omega_{\Lambda}=0.7$ and $H_{0}=70 \mathrm{~km} \mathrm{~s}^{-1} \mathrm{Mpc}^{-1}$. We use a Chabrier (2003) Initial Mass Function (IMF), unless otherwise stated. Magnitudes are provided in the $\mathrm{AB}$ system (Oke \& Gunn 1983).

\section{Data}

\subsection{Galaxy selection criteria}

We retrieve our sample from the GAMA-II database, a panchromatic galaxy survey providing a set of spectroscopic redshifts down to $r_{\mathrm{AB}}=19.8 \mathrm{mag}$ (Liske et al. 2015). We focus on the equatorial fields, that cover $179.94 \mathrm{deg}^{2}$ in three separate regions (see Table 1 in Baldry et al. 2018), with a high ( 98.5\%) spatially uniform redshift completeness that makes it optimal for studies of environment (see, e.g. Robotham et al. 2011; Brough et al. 2013). We note that in GAMA the same fields were repeatedly visited, so that, by construction, the spectroscopic completeness is very high, not only in general, but also over small scales, avoiding the standard issues found in SDSS spectroscopic data sets regarding fibre collision. The tiling and observing strategies of the survey are discussed in detail in Robotham et al. (2010) and Driver et al. (2011).

Star-galaxy separation is described in Baldry et al. (2010). The method notably incorporates a selection of marginallyresolved sources by use of near-infrared colours in addition to the usual Sloan Digital Sky Survey (SDSS; Abazajian et al. 2009) profile separator. 
F. Buitrago et al.: Number densities and environments for low-z massive ultra-compact galaxies

Table 1. Basic observational properties of our MUG sample from GAMA.

\begin{tabular}{|c|c|c|c|c|c|c|c|}
\hline CATAID & RA & Dec & $z_{\text {spec }}$ & $\log _{10} M_{\text {stellar }}$ & $\log _{10}\left\langle\Sigma_{1.5}\right\rangle$ & $\log _{10}\left\langle\Sigma_{\mathrm{e}}\right\rangle$ & $\log _{10}\left\langle\rho_{\mathrm{e}}\right\rangle$ \\
\hline 79071 & 219.96358837 & 0.11254047 & 0.1335 & 11.00 & 10.57 & 9.63 & 9.63 \\
\hline 16143 & 217.73607469 & 0.74513527 & 0.1378 & 10.99 & 10.57 & 9.63 & 9.23 \\
\hline 784327 & 178.74986841 & -1.42614761 & 0.1625 & 11.12 & 11.00 & 10.16 & 10.16 \\
\hline 609701 & 176.87118407 & 0.31908043 & 0.1774 & 10.94 & 10.54 & 9.61 & 9.22 \\
\hline 493286 & 220.3298223 & -1.28795637 & 0.1849 & 11.00 & 10.94 & 10.12 & 10.12 \\
\hline 319149 & 213.61987948 & 1.85908419 & 0.1881 & 11.30 & 10.94 & 10.03 & 9.66 \\
\hline 84466 & 178.0554695 & 0.56305098 & 0.1973 & 11.18 & 10.91 & 10.02 & 10.02 \\
\hline 178241 & 179.75149717 & -1.93248107 & 0.2059 & 11.02 & 10.51 & 9.54 & 9.54 \\
\hline 93202 & 219.52825636 & 0.53898409 & 0.2104 & 10.94 & 10.61 & 9.70 & 9.36 \\
\hline 63726 & 216.76230139 & -0.29771 & 0.2223 & 11.06 & 10.77 & 9.87 & 9.55 \\
\hline 765033 & 223.44512218 & 0.14390729 & 0.2360 & 10.95 & 10.61 & 9.69 & 9.33 \\
\hline 4220443 & 181.69997991 & -1.98105929 & 0.2541 & 11.11 & 10.99 & 10.14 & 9.94 \\
\hline 422365 & 130.57463302 & 2.59286311 & 0.2582 & 11.53 & 11.05 & 10.09 & 9.64 \\
\hline 71471 & 184.92124534 & 0.02897459 & 0.2592 & 11.04 & 10.89 & 10.05 & 10.05 \\
\hline 3873542 & 129.17293417 & -1.27914494 & 0.2609 & 11.19 & 10.81 & 9.89 & 9.51 \\
\hline 300986 & 131.463188 & 1.15208397 & 0.2663 & 11.02 & 11.29 & 10.58 & 10.58 \\
\hline 221269 & 185.47490703 & 1.6248479 & 0.2671 & 10.94 & 10.53 & 9.60 & 9.60 \\
\hline 288762 & 180.01770455 & 1.78508059 & 0.2751 & 11.17 & 10.81 & 9.89 & 9.89 \\
\hline 138954 & 184.98809975 & -1.74957511 & 0.2767 & 11.14 & - & - & - \\
\hline 855304 & 131.15298472 & 1.36284576 & 0.2879 & 10.97 & 11.18 & 10.45 & 10.45 \\
\hline 791716 & 181.36721636 & -1.46390455 & 0.2886 & 11.06 & - & - & - \\
\hline 693193 & 223.21201479 & -0.3199314 & 0.2989 & 11.16 & 10.87 & 9.98 & 9.66 \\
\hline 723783 & 223.09000412 & 2.4662566 & 0.1975 & 10.83 & 11.07 & 10.35 & 10.35 \\
\hline 486049 & 218.39660186 & -1.74897733 & 0.2242 & 10.90 & 10.46 & 9.51 & 9.51 \\
\hline 388238 & 139.19204138 & 2.54304803 & 0.2291 & 10.79 & 10.66 & 9.82 & 9.82 \\
\hline 55006 & 180.21098968 & -0.39229633 & 0.2603 & 10.84 & 10.52 & 9.61 & 9.27 \\
\hline 750374 & 214.89578661 & 0.6837029 & 0.2799 & 10.87 & 10.68 & 9.82 & 9.82 \\
\hline 3173601 & 182.42594704 & 1.27935477 & 0.2874 & 10.79 & 10.80 & 10.01 & 10.01 \\
\hline 365375 & 140.28918164 & 2.62257045 & 0.2874 & 10.85 & 10.49 & 9.57 & 9.57 \\
\hline
\end{tabular}

Notes. The division line splits the galaxies with stellar mass greater or lower than $8 \times 10^{10} M_{\odot}$. Columns: (1) GAMA ID, (2) Right Ascension (J2000), (3) Declination (J2000), (4) Spectroscopic redshift, (5) Stellar mass, in units of $\log \left(M_{\odot}\right)$, (6) 2D stellar surface density according to the prescriptions in Barro et al. (2013), in units of $\log \left(M_{\odot} \mathrm{kpc}^{-1.5}\right)$, (7) 2D stellar surface density in units of $\log \left(M_{\odot} \mathrm{kpc}^{-2}\right)$, (8) 3D stellar density in units of $\log \left(M_{\odot} \mathrm{kpc}^{-3}\right)$. The quantities in Cols. (6-8) are averaged within the effective radius.

Our preselection starts by compiling the set of massive galaxies, defined as those with a stellar mass above $8 \times 10^{10} M_{\odot}$, to be able to compare with previous results in the literature. The sample is extracted from version v20 of the catalogue of stellar masses in the GAMA survey (Taylor et al. 2011), and restricted to the $0.02<z<0.3$ redshift range. The adopted redshift is the so-called Z_TONRY parameter, which corrects for the Virgo-cluster infall at low redshift and uses the cosmic microwave background frame at $z>0.03$ (Tonry et al. 2000).

We note that compact massive galaxies, although already scarce at $z=2-3$ (with comoving number densities $\sim 10^{-4} \mathrm{Mpc}^{-3}$; van Dokkum et al. 2010; Conselice et al. 2011; Buitrago et al. 2013; Barro et al. 2013), in the low- $z$ Universe become very rare objects, with comoving number densities around $10^{-6}$ per $\mathrm{Mpc}^{3}$ (Damjanov et al. 2014, 2015; Tortora et al. 2016; Charbonnier et al. 2017). It is thus imperative to make sure that we start with a conservative enough selection criteria, to ensure we do not miss any potential candidates. Therefore, from the sample of massive galaxies in GAMA, we select those with original SDSS-based effective radii $R_{\mathrm{e}}<2 \mathrm{kpc}$ (Kelvin et al. 2012) in any of the best spatial resolution photometric bands we study (i.e. gri). This cut yields a total of 262 candidates. We compile the Kilo-Degree Survey (KiDS; de Jong et al. 2017) and VISTA Kilo-Degree Infrared Galaxy survey (VIKING; Edge et al. 2013) images of this sample, analyzing them with our bespoke profile fitting $\operatorname{code}^{1}$ (see Sect. 2.2). We visually inspect these galaxies, removing those with GAMA ID (CATAID) 373300 and 537226 as their catalogue-based sizes were only representative of their bulge. Our final sample is defined by those objects that, in the new analysis, consistently give small effective radii $\left(R_{\mathrm{e}}<2 \mathrm{kpc}\right)$ in at least 2 bands, thus avoiding single-band fluctuations in the size determination. This selection reduces the sample to 34 galaxies.

Stellar masses were derived according to the methodology defined in Taylor et al. (2011). There, the authors demonstrated that the $(g-i)$ colour is a very good proxy of the stellar mass-to-light ratio $M_{\star} / L_{i}$. Therefore, the best mass results stem from obtaining the galaxy $i$-band magnitude free from any systematics and then multiplying by its corresponding mass-tolight ratio. In our case, we can estimate more accurately the $i$ band total magnitudes of our MUG sample, as we make use of deep KiDS imaging, in contrast to the SDSS imaging from the original estimates. We modelled the surface brightness profiles with Sérsic functions, not restricted to the detection region. We have thus corrected all the GAMA stellar mass estimates with Sérsic fits (assuming the original mass-to-light ratio) by the formula

$\log M_{\text {new }}=\log M_{\mathrm{GAMA}}+0.4\left(m_{\mathrm{i}, \mathrm{GAMA}}-m_{\mathrm{i}, \mathrm{new}}\right)$,

1 Our profile fitting algorithms can be found at https://github. com/fbuitrago/Profile-fitting 


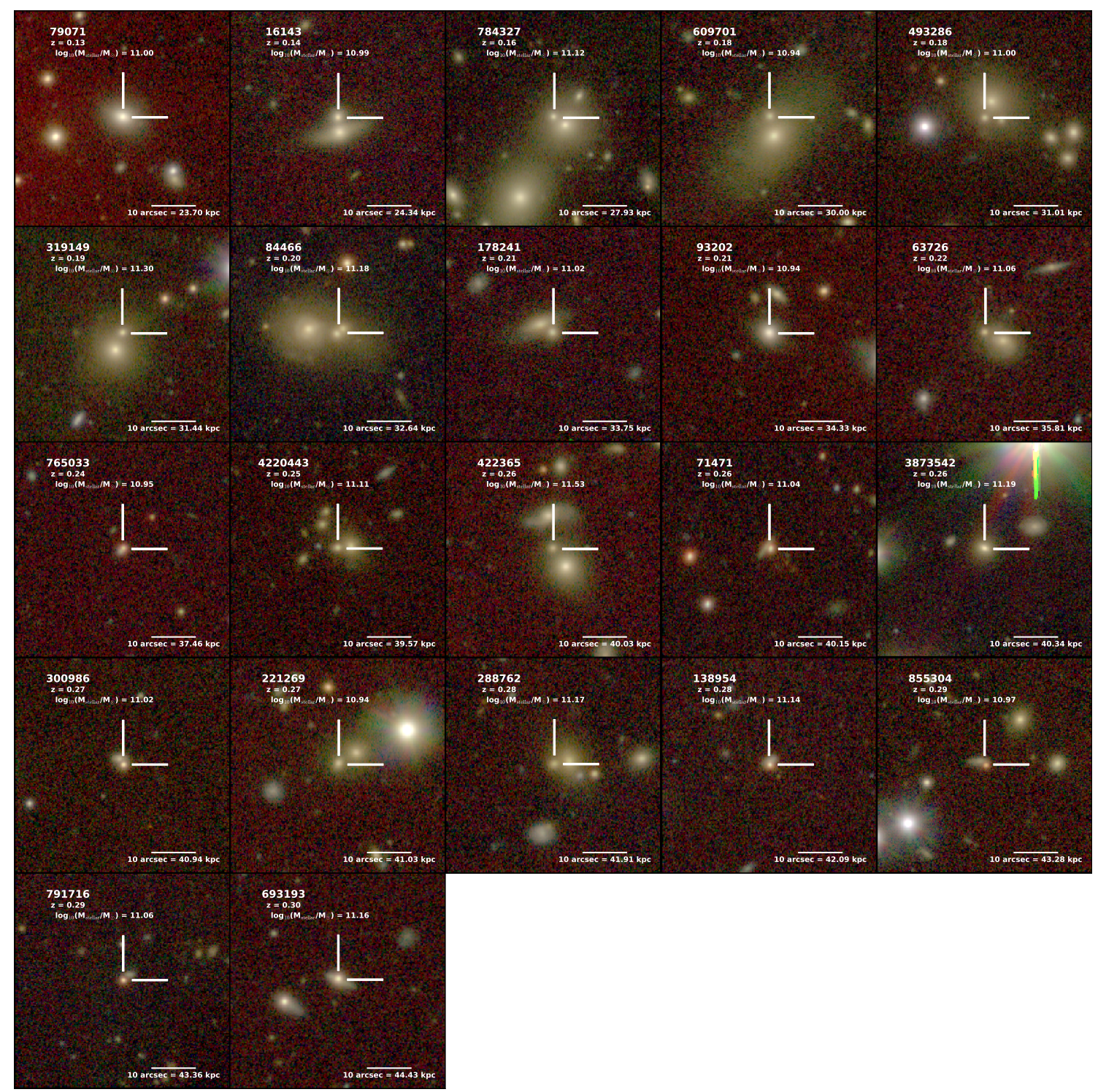

Fig. 1. Mosaic with the $50 \times 50 \operatorname{arcsec}^{2}$ individual images of the 22 galaxies composing our $M_{\star}>8 \times 10^{10} M_{\odot}$ sample. Colour-composite RGB images are created with the $i$-band (red); $r$-band (green) and $g$-band (blue) images from KiDS. The markers pinpoint the exact position of the target galaxy. The surface brightness ranges between 18 and 27 mag arcsec ${ }^{-2}$ in each band. The galaxy name, its spectroscopic redshift and its stellar mass are shown in each image, along with a scale bar with a physical scale.

where $M$ denotes stellar mass and $m$ denotes galaxy magnitude. Hence, our detailed light modelling also improves the mass estimates. In general, the change is not significant, although for some galaxies the stellar masses become smaller due to the fact that we remove the light contribution from neighbouring sources. Hereafter, we use these new, more accurate mass estimates.

As a consequence, 5 objects are rejected from our sample. Our final selection comprises 22 galaxies with effective radii smaller than $2 \mathrm{kpc}$ in at least 2 bands, stellar mass $\geq 8 \times 10^{10}$ and redshift $0.02<z<0.3$. Figure 1 shows a mosaic with the RGB (KiDS) images of our sample of compact massive galaxies. Another 7 galaxies fulfil the size criterion and, given the mass determination uncertainties $(\sim 0.3 \mathrm{dex})$, could potentially enter in our sample. Moreover, we note that compact relics feature bottom-heavy IMFs that could potentially increase the standard IMF-based M/L by a similar amount (Martín-Navarro et al 2015). This additional set appears in Fig. 2, and we will show them separately henceforth.

\subsection{Structural parameter determination}

We cut 50 arcsec postage stamp images in the KiDS $g_{-}, r$ - and $i$-bands and the VIKING Z-band. We fit single Sérsic (1968) functions to the surface brightness profiles of the galaxies in our photometric images. We applied the same procedures developed in Buitrago et al. (2008, 2013). In short, this pipeline first detects the objects in each image applying SExtractor (Bertin \& Arnouts 1996), and then uses GALFIT (Peng et al. 2002, 2010), a code that convolves the 2D galaxy Sérsic models with the PSF of the images and determines the best fit by comparing the convolved model with the observed galaxy surface brightness distribution using a Levenberg-Marquardt algorithm to minimize the $\chi^{2}$ of the fit. The representative PSFs were obtained by the standard procedure of taking isolated nonsaturated stars within our imaging and also from the shapeletbased models for weak lensing developed in Kuijken et al. (2015). Neighbouring objects were masked or fitted along with the target galaxy depending on their proximity. At the end of this 


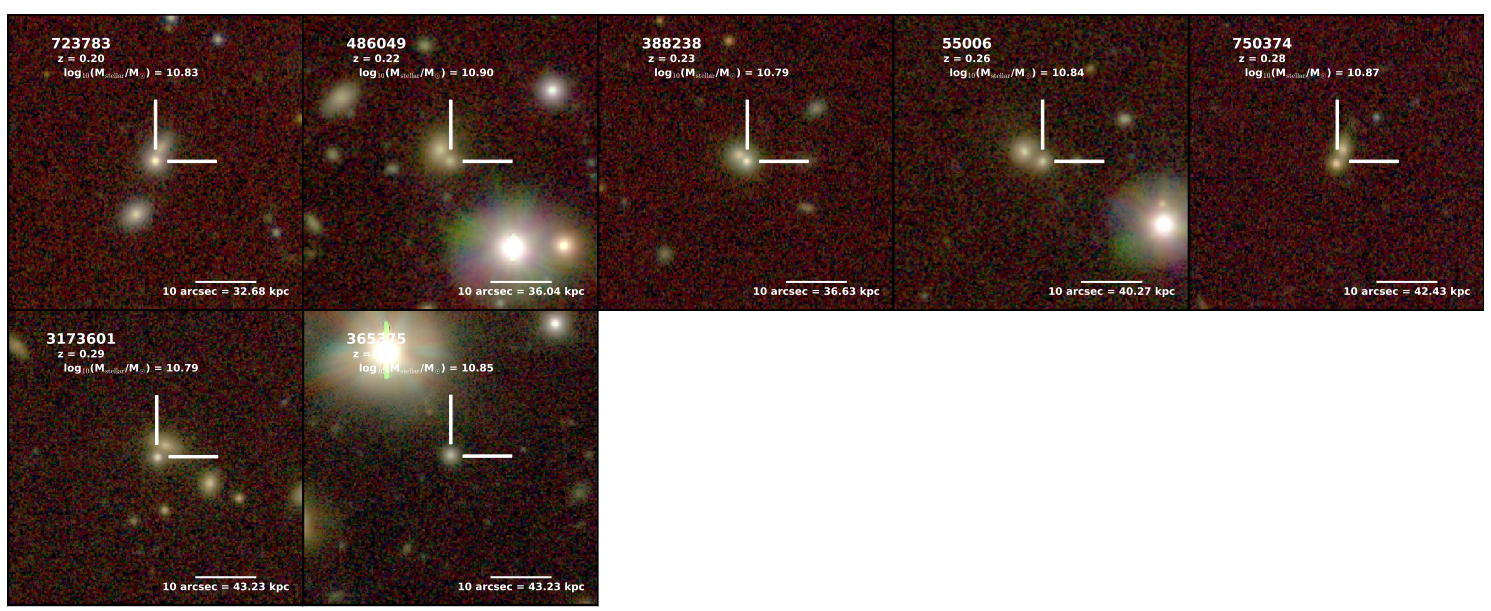

Fig. 2. Mosaic with the $50 \times 50 \operatorname{arcsec}^{2}$ individual images of the 7 galaxies that display effective radii less than $2 \mathrm{kpc}$ in two bands but with stellar masses between $6 \times 10^{10} M_{\odot}$ and $8 \times 10^{10} M_{\odot}$. The RGB stamps are created with the $i$-band (red), $r$-band (green) and $g$-band (blue) images from KiDS. The markers pinpoint the exact position of the target galaxy. The surface brightness levels extend from 18 to $27 \mathrm{mag} \mathrm{arcsec}^{-2}$ in each band. The galaxy name, its spectroscopic redshift and its stellar mass are shown in each image, along with a scale bar with the physical scale.

process, each galaxy was fitted with 4 different PSFs in order to account for the variability of this parameter, taking as our final result the one that displayed the best $\chi^{2}$ value. An example of our fits is shown in Fig. 3.

Since we have access to the PSFs utilized by the weak lensing community, we can see how optimal they are to compute galaxy sizes applying our algorithms. The shapelet-based PSF was the chosen one for $3 / 6 / 5 / 3$ galaxies in the $g / r / i / Z$ bands (i.e. in $10 \%, 21 \%, 17 \%, 10 \%$ of the cases for our $22+7$ object sample). If we only take the shapelet-based PSFs, the sizes deviate on average $\left(3 \sigma\right.$ clipped mean of the quantity $\left(r_{\mathrm{e}, \text { shapelets }}-\right.$ $\left.r_{\text {e,best } \chi^{2}}\right) / r_{\text {e,best }} \chi^{2}$ ) by $-25 \% /-10 \% / 4 \% /-30 \%$. Hence, it seems that the automatic pipelines for weak lensing measurements could be trusted in order to obtain galaxy preselections based on galaxy sizes that will later be refined by the galaxy evolution community.

We conducted several tests to prove the robustness of our structural parameters. In the first instance, we simulated, using again GALFIT, 2D Sérsic functions convolved with the PSFs of the images and injected them into the science images. We add a full description of this procedure in Appendix A. In brief, the results produce realistic errors for the structural parameters and tell us that caution must be applied to results derived from faint and concentrated (high Sérsic index) objects. Additionally, we compare in Fig. 4 the effective radii derived from the deep KiDS and VIKING imaging with previous results for the same galaxies, based on the shallower SDSS data (Kelvin et al. 2012). It is clear that there is little (if any) correlation between these size estimates, with SDSS featuring a wider range of effective radii. This result is expected as the SDSS images are shallower ( $\sim 2$ mag with respect to KiDS; $\sim 1.5$ mag relative to VIKING), and have a coarser sampling (0.396 $\operatorname{arcsec}_{\text {pix }}{ }^{-1}$ in SDSS versus $0.21 / 0.339 \operatorname{arcsec}$ pix $^{-1}$ in KiDS and VIKING respectively), implying uncertain effective radii measurements particularly for such small galaxies.

The structural parameters of our sample are shown in Table 2 ( $r$-band, best seeing band), and also in Table B. 1 for the $g$-band, Table B.2 for the $i$-band and Table B.3 for the $Z$-band. Objects that produced non-valid fits, meaning that their fits are in the constraints of our analysis, are excluded from both the tables and the plots. The effective radii are also shown graphically in Fig. 5 for the 22 object sample and in Fig. 6 for the extra 7 objects. Interestingly, these plots show that for most of the galaxies, there is little variation in the effective radius for all bands, with some exceptions preferentially in the bluest band where the light distribution could be slightly patchier (Buitrago et al. 2008).

\section{Results}

\subsection{The size-mass relation}

Central to the problem of the evolution of massive galaxies is the quantification of the compactness of their stellar component. There has been a range of potential definitions. Several key factors must be taken into account:

- Threshold in mass, size and/or stellar density;

- Photometric band (i.e. rest-frame wavelength) used in the derivation of galaxy sizes;

- Depth of the observations;

- How the sizes are inferred (Sérsic function effective radius, SExTRACTOR-derived half light radius, non-parametric fit radius, number and assumed values for the structural parameter components, e.g. fixing or not their Sérsic index);

- Use of circularized or semi-major axis "radii".

Figure 7 displays the mass-size relation of our sample for all the bands at study, split with respect to morphology, between diskand spheroid-like (Sérsic index $n<2.5$ and $n>2.5$, respectively). The local SDSS size-mass relations (Shen et al. 2003; and their scatter) are overplotted, derived from circularized $z$ band effective radii. If we correct these relations to match our semi-major axis measurements (i.e. non-circularized), they will shift slightly to higher values, matching closer the trends shown with dashed lines, that correspond to the relation inferred for GAMA (Lange et al. 2015). The GAMA relations we show are the ones corresponding to each photometric band, with the same analytical parametrizations for disk- and spheroid-like objects as those in Shen et al. (2003).

It is clear that the objects in our sample feature smaller sizes (at the 2-3 $\sigma$ level) than those expected in galaxies with the same stellar mass in the local relation. Given the large area covered by the observations, we cannot quote a reference value of the PSF FWHM, but the KiDS documentation states that the $r$-band (6231 $\AA$ pivot wavelength) data features the smallest 


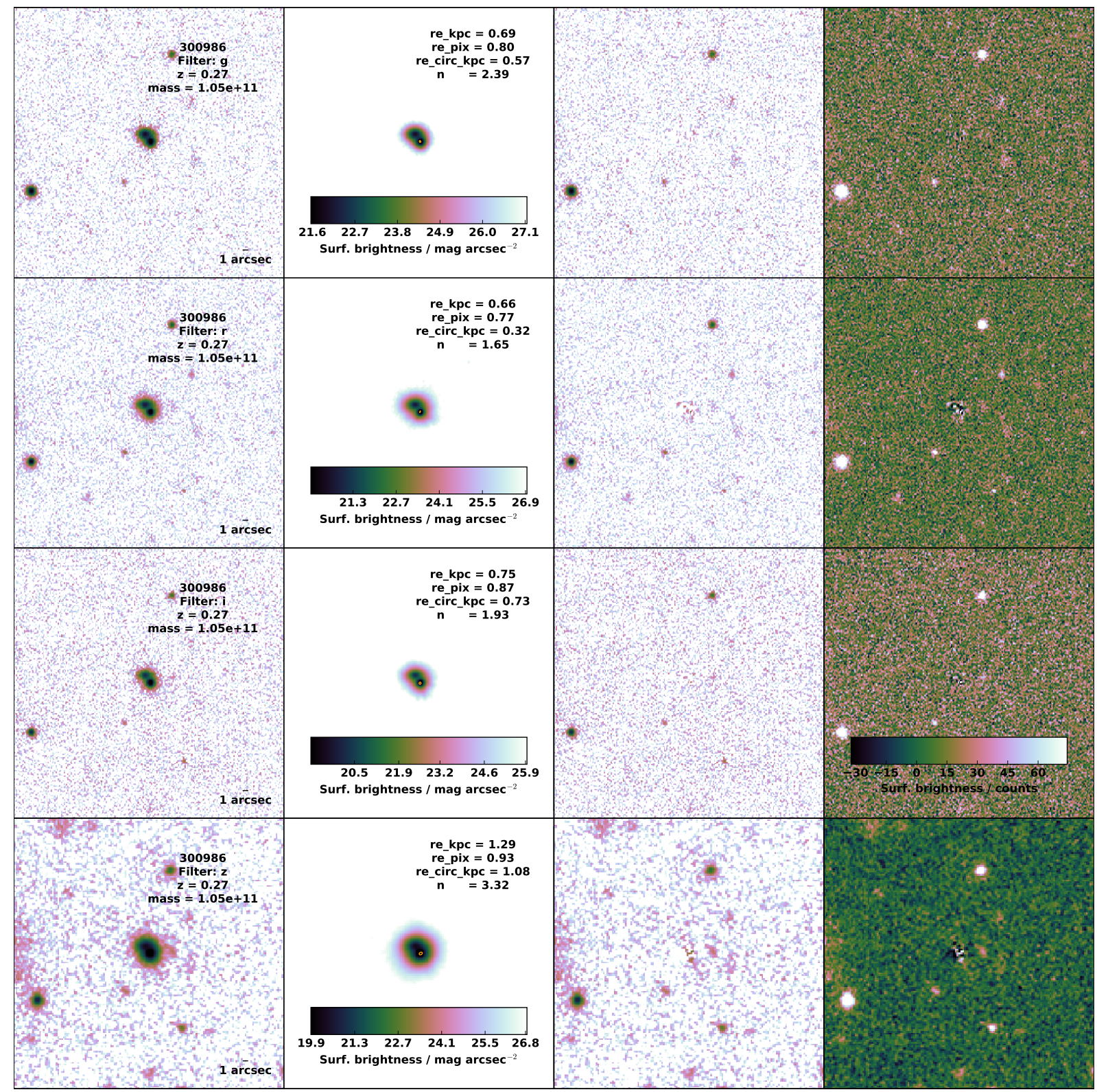

Fig. 3. Results from our single Sérsic fitting analysis for galaxy CATAID 300986. The rows correspond to the photometric bands: (from top to bottom) KiDS $g$, KiDS $r$, KiDS $i$, and VIKING $Z$. From left to right columns: the original $50 \times 50 \operatorname{arcsec}^{2}$ image, its GALFIT model with its associated effective radius ellipse, the residual image (original - model) -in units of surface brightness mag arcsec ${ }^{2}$, that is the reason why one colour bar per band- and the residual image again in units of counts with a single colour bar which is shared by all the images in the last column.

seeing ( $\leq 0.7$ arcsec). Therefore, we will take the $r$-band as the reference filter throughout this paper (rest-frame $\sim 5500-5000 \AA$ for the galaxies in our sample). The $r$-band mass-size relation features the lowest scatter and smallest sizes, reassuring us in the compactness of our galaxy sample. Conversely, we could attribute at least part of the scatter of the galaxy sizes in other bands to the worse seeing conditions, specially taking into account the small size variations expected in massive galaxies, which are usually quiescent objects at $z<0.3$ (Cassata et al. 2010, 2011).

\subsection{On the Sérsic index values for our sample}

Figure 8 displays the Sérsic index values in the $r$-band for the $22+7$ MUGs in our sample. This morphological information is especially valuable at the redshifts covered by our sample. At lower redshift, the galaxy components and traits usually allow an easy visual characterization, while the small (physical and apparent) sizes of the target galaxies - and hence very few pixels subtended in the detector - hinder a robust visual classification of morphology. Half of the 22 objects in the main sample are disk-like and the other half are spheroid-like, although there is a peak at $n=$ $1.5-2$, corresponding to the early disk morphologies seen both at low- and high-z by the Hubble Space Telescope (van der Wel et al. 2011; Buitrago et al. 2013; Trujillo et al. 2014; Ferré-Mateu et al. 2017) and also with ground-based Adaptive Optics observations (Carrasco et al. 2010; Trujillo et al. 2012; Stockton et al. 2014). Interestingly, note that the additional set of 7 lower mass galaxies only features one spheroid-like galaxy (as measured in the $r$-band). 


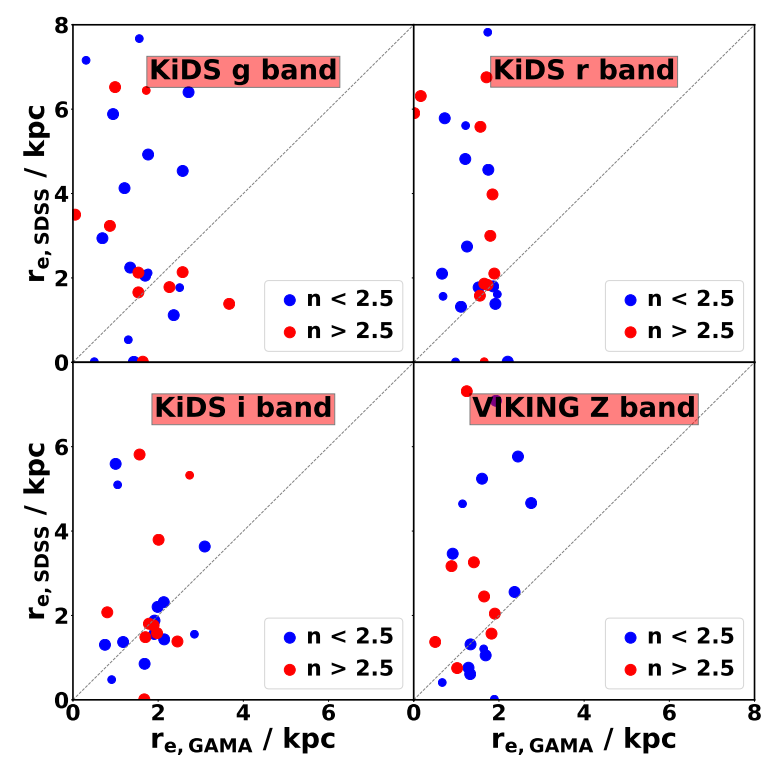

Fig. 4. Inferred effective radii in KiDS ( $g_{-}, r$ - and $i$-bands) and VIKING imaging ( $Z$-band) versus the effective radii in SDSS (as described in Kelvin et al. 2012) for the $22+7$ objects in our sample. The colour coding follows the Sérsic index, with blue dots being disk-like $(n<2.5)$ objects and red dots being spheroid-like $(n>2.5)$ objects. The symbol sizes indicate stellar mass, with big symbols representing $>8 \times 10^{10} M_{\odot}$ galaxies, and small symbols between $6 \times 10^{10} M_{\odot}$ and $8 \times 10^{10} M_{\odot}$. There is little correlation between sizes in the two axes, perhaps only for small galaxies in SDSS being also small in the deeper imaging.

\section{3. $2 D$ and $3 D$ stellar densities}

We show the stellar densities for the objects in our main sample in Fig. 9 derived from the effective radii in the $r$-band, as this is the photometric band with the best seeing $(\leq 0.7 \operatorname{arcsec})$. The solid histogram represents the objects with $M_{\star}>8 \times 10^{10} M_{\odot}$ and the dashed one corresponds to those with $6 \times 10^{10}<$ $M_{\star} / M_{\odot}<8 \times 10^{10}$. The left panel in our plot shows the 2D densities or surface densities within the effective radius and thus the \langle\rangle notation $\left(\left\langle\Sigma_{\mathrm{e}}\right\rangle=M_{\star} / 2 \pi R_{\mathrm{e}}^{2}\right)$. We split our sample between disk-like (blue histogram) and spheroid like objects (red histogram).

We compared our derived densities with those corresponding to the three unambiguously detected relic galaxies (at a distance $\lesssim 106 \mathrm{Mpc}$ away from the Milky Way) from Ferré-Mateu et al. (2017). There, the authors established a "degree of relic", based on the density of the objects and the ages of their stellar populations. The most extreme case is NGC 1277, while the galaxy that appears closer to normal is Mrk1216. In Fig. 9, we see that the MUGs in our sample span densities related to all kinds of "degree of relic". There is also a hint towards higher stellar densities in the disk-like subsample. We attempted to further investigate this aspect by performing the Anderson-Darling test (Anderson \& Darling 1952, a nonparametric two-sample statistic, better suited than Kolmogorov-Smirnov for small samples). However, the statistical significance to reject the hypothesis that disk- and spheroid-like objects come from the same distribution is small (75\% if taking the objects with $M_{\star}>8 \times 10^{10} M_{\odot}, 66 \%$ for those with $M_{\star}>6 \times 10^{10} M_{\odot}$ ).

To shed more light upon this issue, we show 3D stellar densities within the effective radius for our objects in the right panel of Fig. 9, also displaying with different colors disk-like $\left(\left\langle\rho_{\mathrm{e}}\right\rangle=M_{\text {stellar }} / 2 \pi R_{\mathrm{e}}^{2} \mathrm{~h}\right)$ or spheroid-like $\left(\left\langle\rho_{\mathrm{e}}\right\rangle=3 M_{\text {stellar }} / 8 \pi R_{\mathrm{e}}^{3}\right)$ morphology. We did similarly for the three local relics from the literature. In all cases (our sample and the three local galaxies) we assume a disk scale-length of $h=1 \mathrm{kpc}$. The AndersonDarling test now suggests a cleaner separation, rejecting the null hypothesis that disk- and spheroid-like objects come from the same distribution at a $99.5 \%$ significance level for galaxies with $M_{\star}>8 \times 10^{10} M_{\odot}$ and at a $99.9 \%$ significance level for galaxies with $M_{\star}>6 \times 10^{10} M_{\odot}$. Nevertheless, these are only tentative results, as we remind the reader about our small number statistics (only 20 objects) and the strong assumptions regarding the 3D densities (i.e. assuming either a perfect disk - no bulge, no arms, no halo - with a fixed scale length or a homogeneous sphere without any triaxiality). One could attempt to improve this situation in the future using a dynamical analysis (e.g. Schwarzschild modelling) of 3D spectroscopic observations.

Finally, in Fig. 10 we show the compactness criterion following the prescription of Barro et al. (2013), that define a density parameter $\left\langle\Sigma_{1.5}\right\rangle=M_{\star} / R_{\mathrm{e}}^{1.5}$. All the MUGs in our sample inhabit the region delimited by $\log \left\langle\Sigma_{1.5}\right\rangle / M_{\odot} \mathrm{kpc}^{-1.5}>10.3$ (thick blue vertical line), confirming the uniqueness of our sample.

\subsection{Stellar population content}

We use the available optical spectroscopy from GAMA to constrain the underlying stellar populations of our sample of massive and compact galaxies. Only one out of the $22+7$ galaxies have an SDSS spectrum (79071). We note that an additional set of 9 MUGs apparently had a nearby SDSS spectrum, but, on inspection, corresponded to a nearby massive galaxy, as many of our galaxies were found to lie in close pairs or small groups (see Fig. 1). This issue is worth mentioning in the light of the expected incompleteness of the SDSS spectroscopic survey. Another galaxy (93202) only has a low signal-to-noise ratio (S/N) spectrum from the 2dFGRS (Colless et al. 2001) and cannot be used for the analysis of populations. The remaining 20 have spectra from the AAT/AAOmega spectrograph, as well as the additional subsample of 7 potential MUGs, although from this subsample, galaxy 3173601 has a $\mathrm{nQ}=2$ quality flag, meaning the probability of a correct redshift is only $80 \%$.

Unfortunately, the data have a rather low $\mathrm{S} / \mathrm{N}$ for line strength work (the average $\mathrm{S} / \mathrm{N}$ in the 5000-5500 $\AA$ rest-frame window is $\sim 10$ per $\AA$ ), so we decided to focus on a targeted set of line strengths, and to compare with SSP-equivalent parameters. The spectra are corrected for foreground extinction, using the colour excess maps of Schlafly \& Finkbeiner (2011), following a standard dust extinction law for the Milky Way (Cardelli et al. 1989), and brought to the rest-frame.

Figure 11 shows the $4000 \AA$ break strength (using the $D_{n}(4000)$ index of Balogh et al. 1999), the Balmer index $\mathrm{H} \delta_{\mathrm{A}}$ (Worthey \& Ottaviani 1997), and the metallicity-sensitive index [MgFe]' of Thomas et al. (2003). The error bars are given at the $1 \sigma$ level. For reference, we consider $D_{n}(4000)=1.5($ dashed horizontal line) as a threshold to split the sample between "young" and "old" populations. That value of the index corresponds to an SSP-equivalent age of $2 \mathrm{Gyr}$ at solar metallicity. The green lines trace the evolution of the MIUSCAT models (Vazdekis et al. 2012) for a set of simple stellar populations with a Kroupa IMF and solar metallicity, with ages from 0.5 to $10 \mathrm{Gyr}$. The "x" symbols mark the SSP ages 0.5 , 1,2 and 5 Gyr, and the "+" symbols mark the oldest age in the track: $10 \mathrm{Gyr}$. As reference, we include in the figure the results for the sample of massive (stellar mass $>10^{11} M_{\odot}$ ) galaxies from the GAMA survey, with AAT/AAOmega spectra (grey dots). 
Table 2. $r$-band structural parameters of our MUG sample.

\begin{tabular}{|c|c|c|c|c|c|}
\hline CATAID & $\operatorname{mag} \pm \delta \mathrm{mag}$ & $\begin{array}{c}r_{\mathrm{e}} \pm \delta r_{\mathrm{e}} \\
(\mathrm{kpc})\end{array}$ & $\begin{array}{l}r_{\mathrm{e}, \mathrm{circ}} \pm \delta r_{\mathrm{e}, \mathrm{circ}} \\
(\mathrm{kpc})\end{array}$ & $n \pm \delta n$ & $a r \pm \delta a r$ \\
\hline 79071 & $16.93 \pm 0.01$ & $1.92 \pm 0.01$ & $1.67 \pm 0.11$ & $2.08 \pm 0.05$ & $0.76 \pm 0.10$ \\
\hline 16143 & $18.44 \pm 0.01$ & $1.89 \pm 0.06$ & $1.85 \pm 0.20$ & $5.68 \pm 0.19$ & $0.96 \pm 0.15$ \\
\hline 784327 & $19.38 \pm 0.12$ & $1.21 \pm 0.22$ & $1.08 \pm 0.21$ & $2.19 \pm 0.44$ & $0.80 \pm 0.03$ \\
\hline 609701 & $19.51 \pm 0.09$ & $1.85 \pm 0.46$ & $1.67 \pm 0.57$ & $3.75 \pm 0.75$ & $0.81 \pm 0.14$ \\
\hline 493286 & $19.99 \pm 0.09$ & $1.11 \pm 0.07$ & $1.04 \pm 0.25$ & $1.73 \pm 0.62$ & $0.88 \pm 0.30$ \\
\hline 319149 & $19.34 \pm 0.05$ & $1.73 \pm 0.22$ & $1.33 \pm 0.35$ & $4.12 \pm 0.46$ & $0.59 \pm 0.16$ \\
\hline 84466 & $19.22 \pm 0.19$ & $1.52 \pm 0.24$ & $1.45 \pm 0.39$ & $1.62 \pm 0.82$ & $0.91 \pm 0.20$ \\
\hline 178241 & $19.26 \pm 0.40$ & $2.21 \pm 0.46$ & $2.08 \pm 0.48$ & $1.64 \pm 1.13$ & $0.89 \pm 0.03$ \\
\hline 93202 & $17.67 \pm 0.01$ & $1.66 \pm 0.01$ & $1.42 \pm 0.09$ & $5.11 \pm 0.09$ & $0.74 \pm 0.10$ \\
\hline 63726 & $19.91 \pm 0.05$ & $1.57 \pm 0.19$ & $1.39 \pm 0.46$ & $4.03 \pm 0.45$ & $0.79 \pm 0.33$ \\
\hline 765033 & $19.86 \pm 0.01$ & $1.71 \pm 0.01$ & $0.80 \pm 0.17$ & $6.07 \pm 0.13$ & $0.22 \pm 0.09$ \\
\hline 4220443 & $19.96 \pm 0.01$ & $1.21 \pm 0.27$ & $0.67 \pm 0.30$ & $4.66 \pm 1.10$ & $0.31 \pm 0.13$ \\
\hline 422365 & $19.94 \pm 0.10$ & $2.11 \pm 0.53$ & $1.99 \pm 0.66$ & $3.15 \pm 0.63$ & $0.89 \pm 0.15$ \\
\hline 71471 & $19.34 \pm 0.09$ & $1.25 \pm 0.08$ & $1.16 \pm 0.14$ & $0.92 \pm 0.33$ & $0.86 \pm 0.09$ \\
\hline 3873542 & $18.63 \pm 0.02$ & $1.80 \pm 0.06$ & $1.21 \pm 0.36$ & $3.21 \pm 0.08$ & $0.45 \pm 0.24$ \\
\hline 300986 & $19.43 \pm 0.09$ & $0.66 \pm 0.04$ & $0.32 \pm 0.11$ & $1.65 \pm 0.59$ & $0.23 \pm 0.13$ \\
\hline 221269 & $19.63 \pm 0.20$ & $1.86 \pm 0.30$ & $1.37 \pm 0.56$ & $1.16 \pm 0.59$ & $0.54 \pm 0.27$ \\
\hline 288762 & $20.01 \pm 0.09$ & $1.75 \pm 0.11$ & $1.11 \pm 0.26$ & $1.61 \pm 0.58$ & $0.40 \pm 0.14$ \\
\hline 138954 & $19.28 \pm 0.03$ & - & - & - & - \\
\hline 855304 & $20.93 \pm 0.09$ & $0.73 \pm 0.05$ & $0.35 \pm 0.07$ & $1.40 \pm 0.50$ & $0.23 \pm 0.07$ \\
\hline 791716 & $20.10 \pm 0.01$ & - & - & - & - \\
\hline 693193 & $18.22 \pm 0.01$ & $1.55 \pm 0.03$ & $1.43 \pm 0.31$ & $5.50 \pm 0.05$ & $0.85 \pm 0.34$ \\
\hline 723783 & $18.21 \pm 0.08$ & $0.69 \pm 0.13$ & $0.44 \pm 0.20$ & $0.83 \pm 0.11$ & $0.41 \pm 0.21$ \\
\hline 486049 & $19.70 \pm 0.20$ & $1.96 \pm 0.31$ & $1.83 \pm 0.52$ & $1.40 \pm 0.71$ & $0.87 \pm 0.22$ \\
\hline 388238 & $18.66 \pm 0.09$ & $1.22 \pm 0.23$ & $1.14 \pm 0.37$ & $1.82 \pm 0.23$ & $0.87 \pm 0.24$ \\
\hline 55006 & $19.43 \pm 0.01$ & $1.65 \pm 0.37$ & $1.43 \pm 0.59$ & $4.34 \pm 1.02$ & $0.75 \pm 0.29$ \\
\hline 750374 & $19.75 \pm 0.09$ & $1.34 \pm 0.09$ & $1.24 \pm 0.20$ & $1.30 \pm 0.46$ & $0.86 \pm 0.17$ \\
\hline 3173601 & $19.54 \pm 0.09$ & $0.98 \pm 0.06$ & $0.72 \pm 0.13$ & $1.98 \pm 0.71$ & $0.54 \pm 0.12$ \\
\hline 365375 & $20.05 \pm 0.09$ & $1.74 \pm 0.11$ & $1.68 \pm 0.01$ & $0.92 \pm 0.33$ & $0.93 \pm 0.01$ \\
\hline
\end{tabular}

Notes. The division line splits the galaxies with stellar mass greater or lower than $8 \times 10^{10} M_{\odot}$. Columns: (1) GAMA ID, (2) magnitude, (3) effective radius in kpc, (4) circularized effective radius in kpc, (5) Sérsic index, (6) axis ratio.

In the figure, the size of the symbols split the sample between the bona-fide set (large dots) and the additional 7, lower-mass candidates (smaller dots). The dots are also colour-coded - blue for disk-like $(n<2.5)$ objects and red for spheroid-like systems $(n>2.5)$. Although the $\mathrm{S} / \mathrm{N}$ is not high enough for a detailed analysis of the populations, our sample features a wide range of ages, with 7 galaxies out of $29(\sim 25 \%)$ having older populations (therefore "relics"), where both $D_{n}(4000)$ and $\mathrm{H} \delta_{\mathrm{A}}$ suggest old ages. These relics are split into four disk-like systems (79071, 221269, 84466 and 486049) and 3 spheroids (3873542, 319149 and 609701). Out of the four disks, only one galaxy lacks an indication of a galactic companion (79071), and also one of the spheroids (3873542) appears as an isolated galaxy. We will explore the environment of our MUGs in more detail in Sect. 3.5. The galaxy with the strongest $4000 \AA$ break (319149) is expected to have a very high formation redshift $\left(z_{\mathrm{FOR}} \gtrsim 3\right)$. It resembles the local relic NGC 1277: it is a very massive $\left(M_{\star}=2 \times 10^{11} M_{\odot}\right)$ satellite of an even more massive galaxy. Apart from these aspects, this plot shows no clear trend between galaxy morphology and mass with respect to the spectral features.

Figure 12 shows the UVJ diagram, with divisions as defined in Williams et al. (2009), which has proved to be a powerful tool to photometrically discern quiescent (red region) from dusty star forming (blue region) galaxies (see, e.g. Díaz-García et al.
2017). Our sample is shown with the same colour- and sizecoding as in Fig. 11. The rest-frame colours are derived from comparisons of the observed $D_{n}(4000), \mathrm{H} \delta_{\mathrm{A}}$ and $[\mathrm{MgFe}]^{\prime}$ line strengths with a grid of simple stellar populations from the models of Bruzual \& Charlot (2003), covering a range of ages (from 0.5 to $13 \mathrm{Gyr}$ ) and metallicities (from $[M / H]=-0.3-$ $0.3 \mathrm{dex}$ ). The K-corrections were derived from the spectroscopic information, by comparing the observed line strengths with a set of stellar populations, as above. The best fit spectrum is then used to translate between rest-frame $U$ and observed $g$ (KiDS); rest-frame $V$ and observed $r$ (KiDS); and rest-frame $J$ and observed $H$ (VIKING). We note that for K-corrections to stay below a $\sim 5 \%$ level, it is not necessary to apply more detailed analyses of the underlying populations, as the method simply performs a sort of "interpolation" in the best-fit spectra to compute the colour transformations, and, given the redshifts covered, this interpolation is not stretched too much in wavelength. The UVJ colour diagram classifies nine objects as quiescent, namely the spheroids (3873542, 609701, 16143, 422365 and 693193) and the disks (288762, 486049, 723783 and 79071). However, a fair amount of scatter is also evident throughout the whole sample, with objects deeply in the star-forming region. Part of the (horizontal) observed scatter in this diagram may be due to the fact that the $H$-band imaging is shallower, with poorer resolution. 


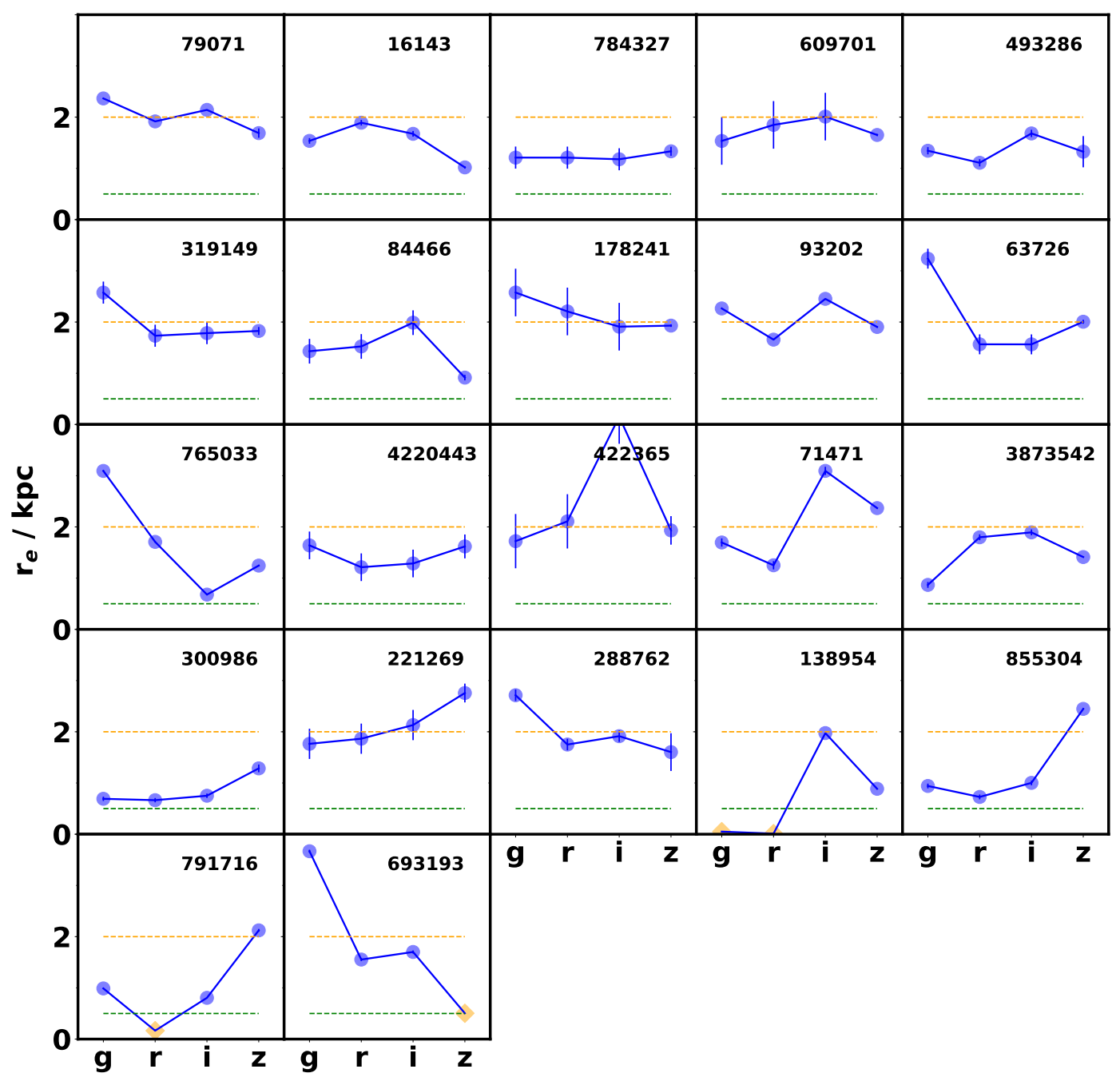

Fig. 5. Mosaic displaying the effective radii in each band for the 22 galaxies in our MUG sample. As reference, the horizontal dotted lines correspond to $R_{\mathrm{e}}=0.5 \mathrm{kpc}$ and $R_{\mathrm{e}}=2 \mathrm{kpc}$. Bad fits are denoted by orange diamonds.

\subsection{The role of environment}

Environment is arguably one of the most important factors driving the evolution of compact massive galaxies with redshift. Within the framework of the two-stage formation scenario (e.g. Oser et al. 2010), massive galaxies have an in-situ formation channel, by which gas is typically fed to the centre of the galaxy, triggering a star formation burst, and an ex-situ channel where stars are incorporated into the galaxy via mergers. Minor mergers may be especially relevant in the evolution from massive cores at high redshift to the present massive and large galaxies, as this type of mergers tend to populate the outer regions of galaxies (Naab et al. 2009). This could lead to the formation of a spheroidal envelope (Hopkins et al. 2009; Bezanson et al. 2009) or the growth of a galaxy disk (Graham et al. 2015; de la Rosa et al. 2016), but always resulting in an increased size and a subsequent evolution on the mass-size plane (Buitrago et al. 2017).

Therefore, one should expect the growth of massive galaxies to be strongly dependent on environment, reflecting their past merger history. Valentinuzzi et al. (2010a,b) found a rather high prevalence of massive compact galaxies in low- $z$ clusters, amounting to $\sim 20 \%$ of their total sample of cluster galaxies with stellar mass above $3 \times 10^{10} M_{\odot}$. This result is in stark contrast with the $4.4 \%$ equivalent fraction found in the field (Poggianti et al. 2013). Moreover, the clearest example of a local compact and massive relic is NGC 1277, situated in the Perseus cluster (van den Bosch et al. 2012; Ferré-Mateu et al. 2017). By definition, low-redshift relics should represent galaxies that formed a very massive core early on (at redshift $z \gtrsim$ $2-3$ ), and did not experience any significant subsequent merging - or alternatively underwent a dramatic dynamical event that removed its envelope at a later time. Therefore, we can roughly separate two major channels for the formation of MUGs, based on environment, that will create two different populations: 1) a high density sample representing galaxies that were incorporated into clusters at early times, with two potential scenarios: either systems that did not undergo any merger, given the high relative velocities, or non-compact massive galaxies that were stripped of their outer envelope via tidal interactions in the cluster; and 2) a low density sample, where these galaxies represent local and isolated high-density peaks that allowed for the formation of a massive galaxy at early times, but where the local density was not high enough to experience any mergers. The latter represents a cleaner, but, obviously, a much rarer sample than the former. Our GAMA-based selection is the best option at present to target this question. 


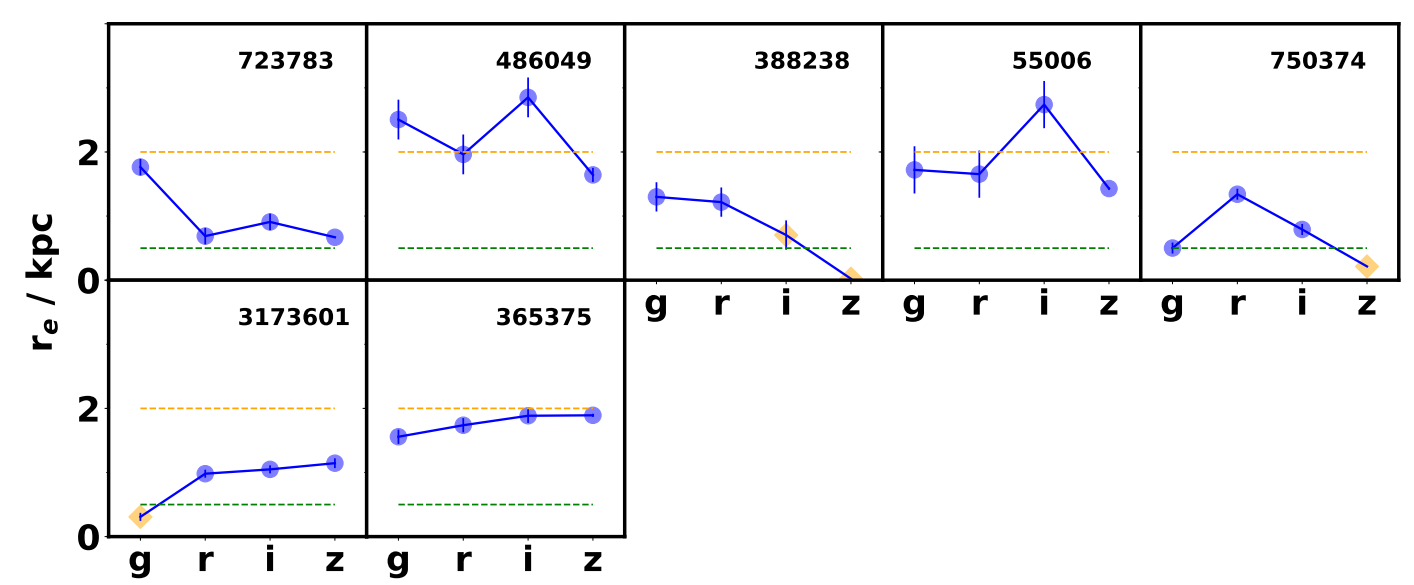

Fig. 6. Mosaic displaying the effective radii in each band from the 7 galaxies that display effective radii less than $2 \mathrm{kpc}$ in two bands but with stellar masses between $6 \times 10^{10} M_{\odot}$ and $8 \times 10^{10} M_{\odot}$. As reference, horizontal dotted lines correspond to $R_{\mathrm{e}}=0.5 \mathrm{kpc}$ and $R_{\mathrm{e}}=2 \mathrm{kpc}$. Bad fits are denoted by orange diamonds.
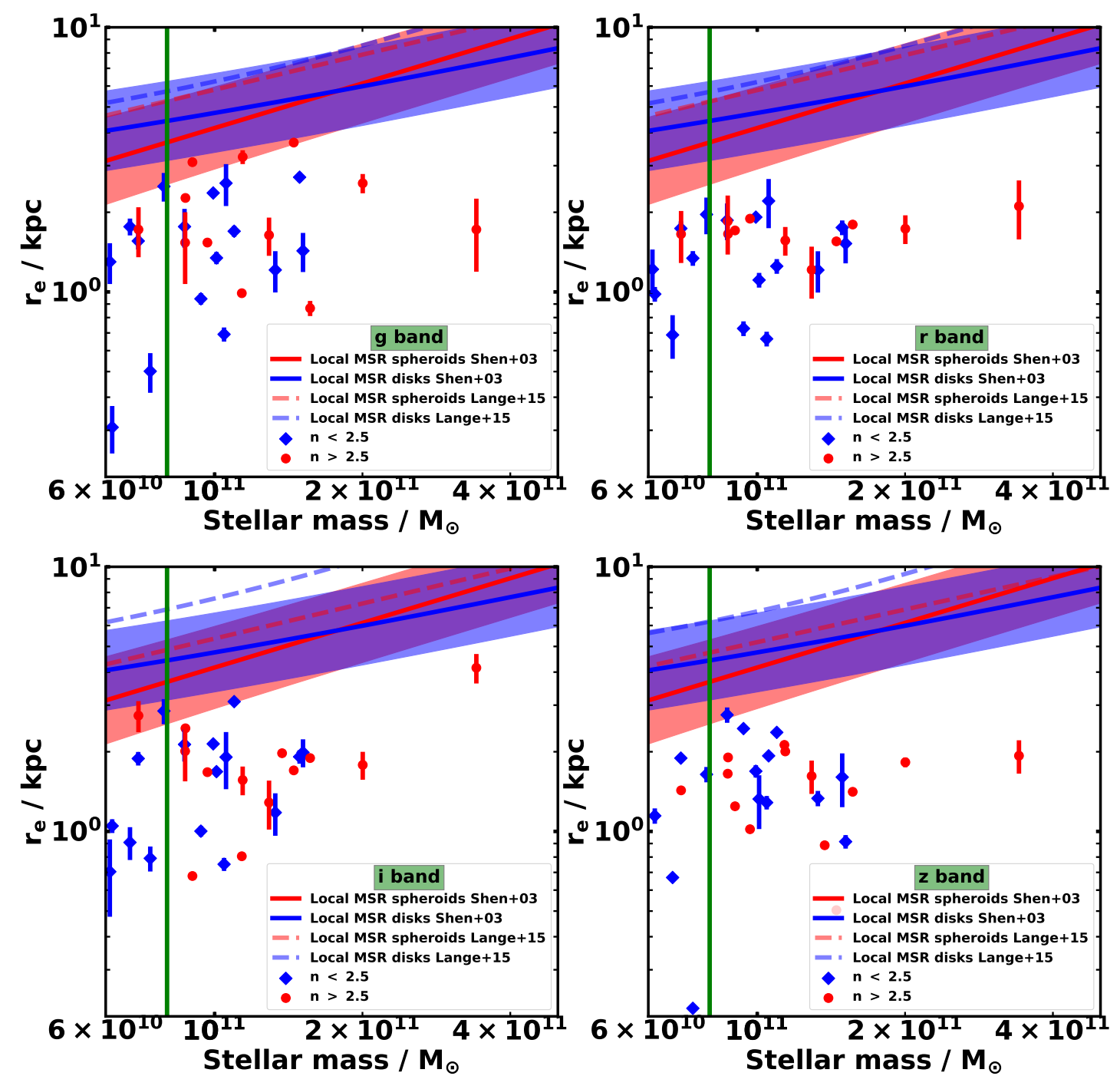

Fig. 7. Mass-size relation in all bands included in our study (from top to bottom, from left to right panels: $g$-, $r$-, $i$ - and $Z$-band). Objects are split with respect to the surface brightness profile into disk-like $(n<2.5$; in blue) and spheroid-like $(n>2.5$; in red). The green vertical line separates the objects in the main sample $\left(M_{\text {stellar }} \geq 8 \times 10^{10} M_{\odot}\right)$ from those that might be compatible if we include the mass uncertainties $\left(M_{\text {stellar }} \geq 6 \times 10^{10} M_{\odot}\right)$. The solid regions represent the canonical SDSS $z$-band mass-size relation from Shen et al. (2003), including the dispersion. The dashed lines are the equivalent trends for GAMA galaxies from Lange et al. (2015), for each photometric band. The error bars are derived according to the results in our simulations. Note the effective radii for our sample are not circularized. 


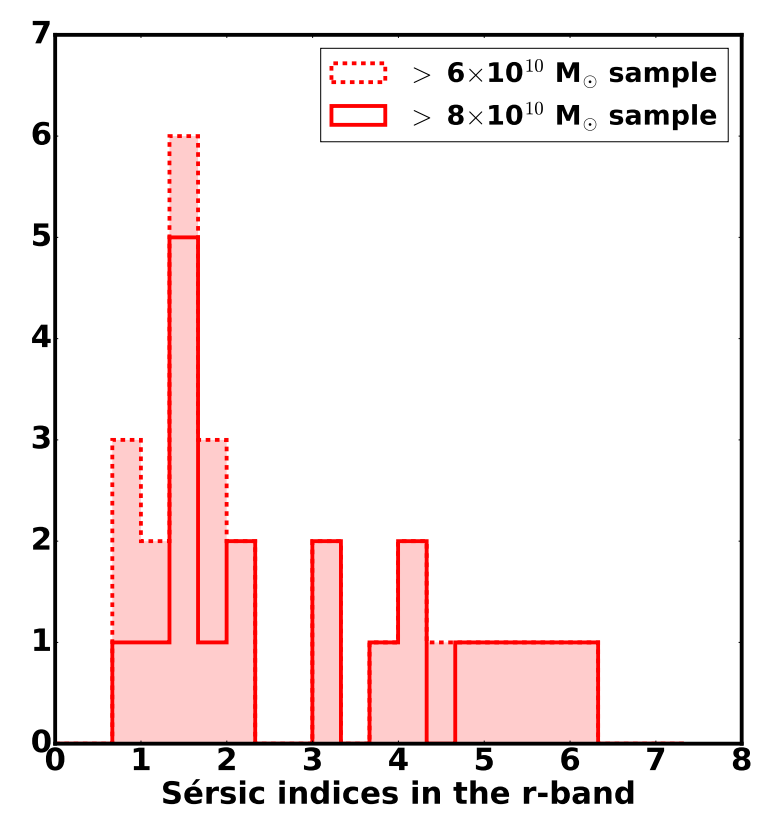

Fig. 8. Histogram of the Sersic indices in the $r$-band - our filter with the best seeing $(\leq 0.7 \mathrm{arcsec})$. The solid histogram corresponds to MUGs with $M_{\star}>8 \times 10^{10} M_{\odot}$ (note that only 20 objects appear as the other 2 have non-valid analyses in the $r$-band) while the dashed ones are for $6 \times 10^{10}<M_{\star} / M_{\odot}<8 \times 10^{10}$ galaxies. This histogram reveals that the majority of the objects are disk-like $(n<2.5)$, in accordance to the "swollen disk" morphology found in relic galaxies and in general for MUGs at low- and high- $z$.

We contrast here our sample with the general distribution of massive galaxies regarding group mass. The GAMA survey was especially designed for the robust determination of galaxy environments, minimising as much as possible the incompleteness of the spectroscopically-derived redshifts. The group masses are taken from the $\mathrm{G}^{3} \mathrm{C}$ catalogue of Robotham et al. (2011, we use $\mathrm{v} 10$ ), following the scaling relation with total luminosity from Viola et al. (2015). Figure 13 shows the comparison, where our massive compact galaxies are shown as filled circles - following the same colour coding as in Fig. 11 - while the Kernel Density Estimation and the individual black dots represent the general population of massive galaxies in GAMA. Only 17/6 of the 22/7 MUGs have a group allocation in the $\mathrm{G}^{3} \mathrm{C}$ catalogue. Most of our sample lies close to their group centre, but they do not reside in the most massive groups of the survey. Only four galaxies are located in groups more massive than $10^{14} M_{\odot}$, representing only $15 \%$ of the total. The GAMA "Environment Measures" catalogue of Brough et al. (2013) only lists 3 of our MUG candidates ${ }^{2}$, namely $79071\left(\Sigma_{5}=2.58 \mathrm{Mpc}^{-2}\right)$; $16143\left(\Sigma_{5}=6.94 \mathrm{Mpc}^{-2}\right)$; and $609701\left(\Sigma_{5}=8.53 \mathrm{Mpc}^{-2}\right)$, where the numbers in brackets give the surface number densities estimated within the 5th nearest neighbour. These kind of densities imply a distance to a 5th neighbour, with absolute magnitude brighter than $M_{r}<-20$, of $<0.8 \mathrm{Mpc}$. We note that in the GAMA sample, the distribution of $\Sigma_{5}$ has a mean of $2.68 \mathrm{Mpc}^{-2}$, and a median of $0.35 \mathrm{Mpc}^{-2}$. Therefore, these three galaxies are clearly located in high density regions, although not at the highest densities expected of galaxy clusters.

Note that given the special character of this sample, and the relatively faint fluxes probed with respect to the GAMA thresh-

This catalogue is limited to $z_{\mathrm{TONRY}}<0.18$. old (see Table 2), we may wonder whether the completeness is high enough for these specific targets. For each detection, GAMA provides a parameter (MASK_IC) that gives the completeness level within a region. In our sample, the completeness levels are in most cases above $90 \%$ except for two sources: 79071 (with 87\% completeness) and 319149 (88\%). How well are we able to detect the host environments of MUGs? A simple estimate based on simple stellar populations from the synthetic models of Bruzual \& Charlot (2003) shows that at the highest redshift of our set $(z \sim 0.3)$, a solar-metallicity, dustless $10 \mathrm{Gyr}$ old population - the oldest possible at that redshift - with stellar mass $10^{11} M_{\odot}$ would have an apparent magnitude $r \sim 19.8 \mathrm{AB}$, which actually is the limiting magnitude of the GAMA survey. Follow-up spectroscopy covering the fainter sources surrounding the MUGs will be needed to understand the issue of environment in more detail.

\subsection{Number densities of ultracompact galaxies at $0.02<z<0.3$}

Figure 14 shows the comoving number densities of MUGs at $0.02<z<0.3$. Our data points are the blue stars, combining both our $M_{\star}>8 \times 10^{10} M_{\odot}$ and our $6 \times 10^{10}<M_{\star} / M_{\odot}<$ $8 \times 10^{10}$ MUG subsamples. The inferred number density values are listed in Table 3. The shaded regions represent the results from Quilis \& Trujillo (2013), where they show the redshift evolution of the number density of massive $\left(>8 \times 10^{10} M_{\odot}\right)$ galaxies whose stellar masses have been modified after their formation episode by less than 10\% (yellow area) and 30\% (red area), according to the simulations in De Lucia \& Blaizot (2007) and Guo et al. (2011, 2013).

We did not find any MUG at $z<0.1$, but we can place an upper limit from our observations and also an additional data point at $z \sim 0$ from the 3 relic galaxies in Ferré-Mateu et al. (2017). However, we note that, according to our definition of compactness, only NGC 1277 and PGC 32873 would be included in our sample (rejecting Mrk1216). We also contrast these results with those in Yildirım et al. (2017), where only 2 galaxies would pass our selection, NGC 1277 and PGC 70520 (discarding PGC 32873 this time). Therefore, broadly speaking, there are 3 galaxies consistent with our definition of a MUG within a distance of $106 \mathrm{Mpc}$, namely NGC 1277, PGC 32873 and PGC 70520; and thus we keep the number density value of $6 \times 10^{-7} \mathrm{Mpc}^{-3}$ at $z \sim 0$ from Ferré-Mateu et al. (2017).

At $z>0$, there is a disparity in the data point values presented in our plot, because of the non-homogeneity in the MUG definitions. We took in all cases the selection criteria that are closest to ours. At $0.2<z<0.3$, we show:

- Charbonnier et al. (2017): $M_{\star}>10^{10.7} M_{\odot}$ and $R_{\mathrm{e}}<$ $1.5 \mathrm{kpc} \cdot\left(M_{\star} / 10^{11} M_{\odot}\right)^{0.75}(i$-band imaging of SDSS/Stripe 82 with the CFHT).

- Damjanov et al. (2014): $M_{\text {dyn }}>8 \times 10^{10} M_{\odot}$ (stellar-like objects in SDSS data, classified as quiescent galaxies, for which dynamical masses are obtained).

- Damjanov et al. (2015): $M_{\star}>8 \times 10^{10} M_{\odot}$ and $R_{\mathrm{e}}<$ $2.5 \mathrm{kpc} \cdot\left(M_{\star} / 10^{11} M_{\odot}\right)^{0.75}(\mathrm{ACS} / \mathrm{COSMOS}$ data of passive galaxies).

- Tortora et al. (2018), superseding Tortora et al. (2016): $M_{\star}>8 \times 10^{10} M_{\odot}$ and $R_{\mathrm{e}, \text { circ }}<1.5 \mathrm{kpc}$ (median between $g-, r$ - and $i$-bands).

Summarizing, the disparity among different works comes from a combination of both slightly different selection criteria and the scarcity of this type of galaxy. Given the uncertainties from the different studies, we could affirm that the number density 


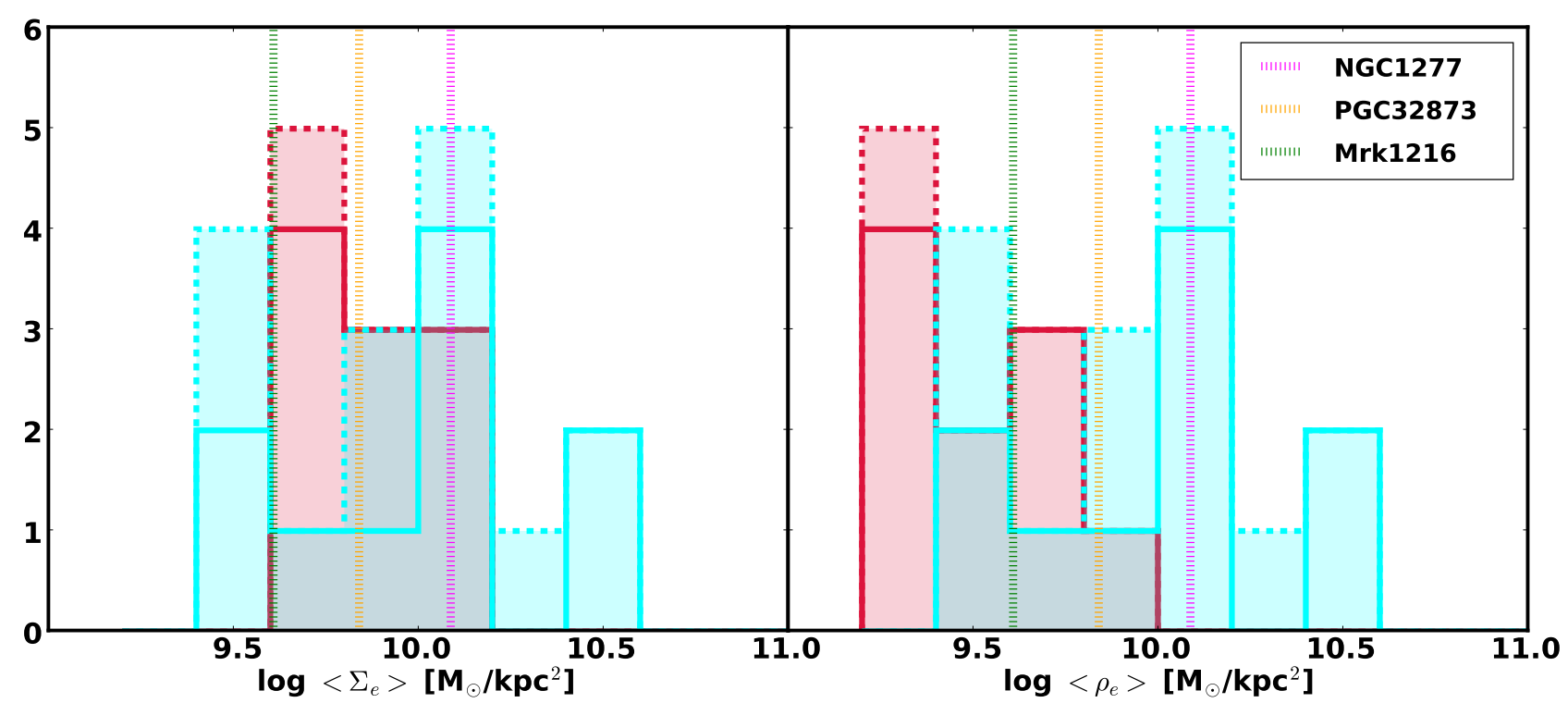

Fig. 9. Left panel: histogram with the $2 \mathrm{D}$ stellar mass density within one effective radius $\left(<\Sigma_{\mathrm{e}}>=M_{\star} / 2 \pi R_{\mathrm{e}}^{2}\right)$ of our MUG sample, split between disk- (blue) and spheroid-like (red) morphology $\left(n<2.5\right.$ and $n>2.5$, respectively). The solid histogram denotes the objects with $M_{\star}>8 \times 10^{10} M_{\odot}$ and the dashed one those with $6 \times 10^{10}<M_{\star} / M_{\odot}<8 \times 10^{10}$. Overplotted, as vertical dotted lines, are the values for the confirmed relic galaxies in Ferré-Mateu et al. (2017). The MUGs in our study span the range of values expected of relic galaxies. Right panel: histogram showing the 3D stellar mass density within one effective radius of our MUG sample, again dividing it between $M_{\star}>8 \times 10^{10} M_{\odot}$ (solid histogram) and $6 \times 10^{10}<M_{\star} / M_{\odot}<8 \times 10^{10}$ (dashed histogram). We split the galaxies into disk- (blue) and spheroid-like (red) morphology $(n<2.5$ and $n>2.5$, respectively). We also compute their densities differently: $\left\langle\rho_{\mathrm{e}}>=M_{\text {stellar }} / 2 \pi R_{\mathrm{e}}^{2} \mathrm{~h}\right.$ for disks (with $h=1 \mathrm{kpc}$ ), and $\left\langle\rho_{\mathrm{e}}>=3 M_{\text {stellar }} / 8 \pi R_{\mathrm{e}}^{3}\right.$ for spheroids. Overplotted are the values of the confirmed local relic galaxies from Ferré-Mateu et al. (2017) - using our formula for disks. On average, disks appear slightly denser than spheroids.

of these objects is $\sim 10^{-6} \mathrm{Mpc}^{-3}$ at $0<z<0.3$. The error bars in our data are consistent with either a flat slope or a weak redshift evolution, as suggested by the coloured areas in Fig. 14.

\section{Conclusions}

We present in this paper a complete census within the footprint of the GAMA survey of massive ultra-compact galaxies (MUGs) at $0.02<z<0.3$, placing special emphasis on their number density and environmental determination. GAMA is the perfect benchmark for this study due to its spatially uniform spectroscopic completeness ( $~ 98.5 \%$ down to $r=19.8 \mathrm{ABmag}$ ) over a large area of the sky $\left(180 \mathrm{deg}^{2}\right)$ with deep ancillary photometric data ( $g$-, $r$ - and $i$-bands from KiDS; $Z$-band from VIKING). This imaging enabled us to produce robust size estimates, as it is $\sim 2$ mag deeper than SDSS and with better spatial resolution $(<0.7$ arcsec seeing in the $r$-band $)$.

Our final sample consists of 22 objects with effective radii $<2 \mathrm{kpc}$ in at least two photometric bands and stellar masses $M_{\star}>8 \times 10^{10} M_{\odot}$. We include an additional set of 7 MUGs if taking into account our mass uncertainties, and a potentially heavier initial mass function within this type of galaxies. This work builds up from previous low-redshift size determinations in SDSS data, conducting a careful analysis of pre-selected compact galaxies, using deeper imaging and simulations to assess the reliability of our results.

MUGs are located, by definition, at the bottom of the galaxy mass-size relation (see Fig. 7), but interestingly they usually display early disk/swollen disk morphologies (Fig. 8) and very large stellar densities (Fig. 9). Some of them host very old and passive stellar populations (Figs. 11 and 12), therefore being "red nugget" galaxies that survive unaffected over a large fraction

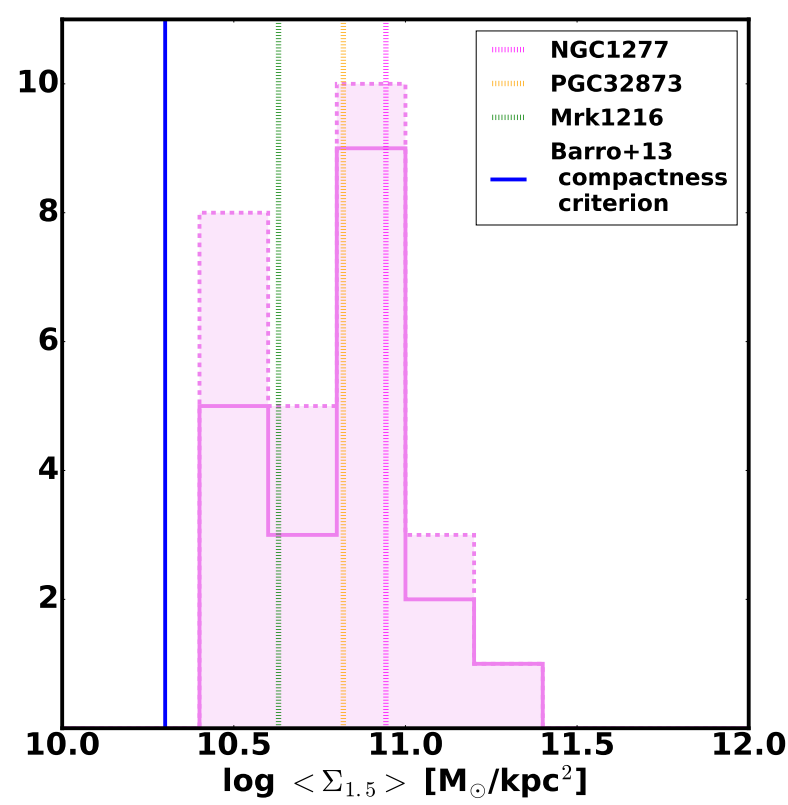

Fig. 10. Distribution of the 2D stellar mass density of our MUGs, following the parameter $\left\langle\Sigma_{1.5}\right\rangle \equiv M_{\star} / R_{\mathrm{e}}^{1.5}$ to define compactness, as in Barro et al. (2013). Overplotted are the values for the confirmed nearby relic galaxies given in Ferré-Mateu et al. (2017). The MUGs in our study span a similar range of density as in relic galaxies, and some even surpass the largest local value of the surface mass density (NGC 1277).

of the present age of the Universe. Hence, the study of this population (at low redshift) is a true window to the early phases of galaxy evolution.

State-of-the-art simulations suggest that relic galaxies should inhabit the central regions of galaxy clusters in order not to have 


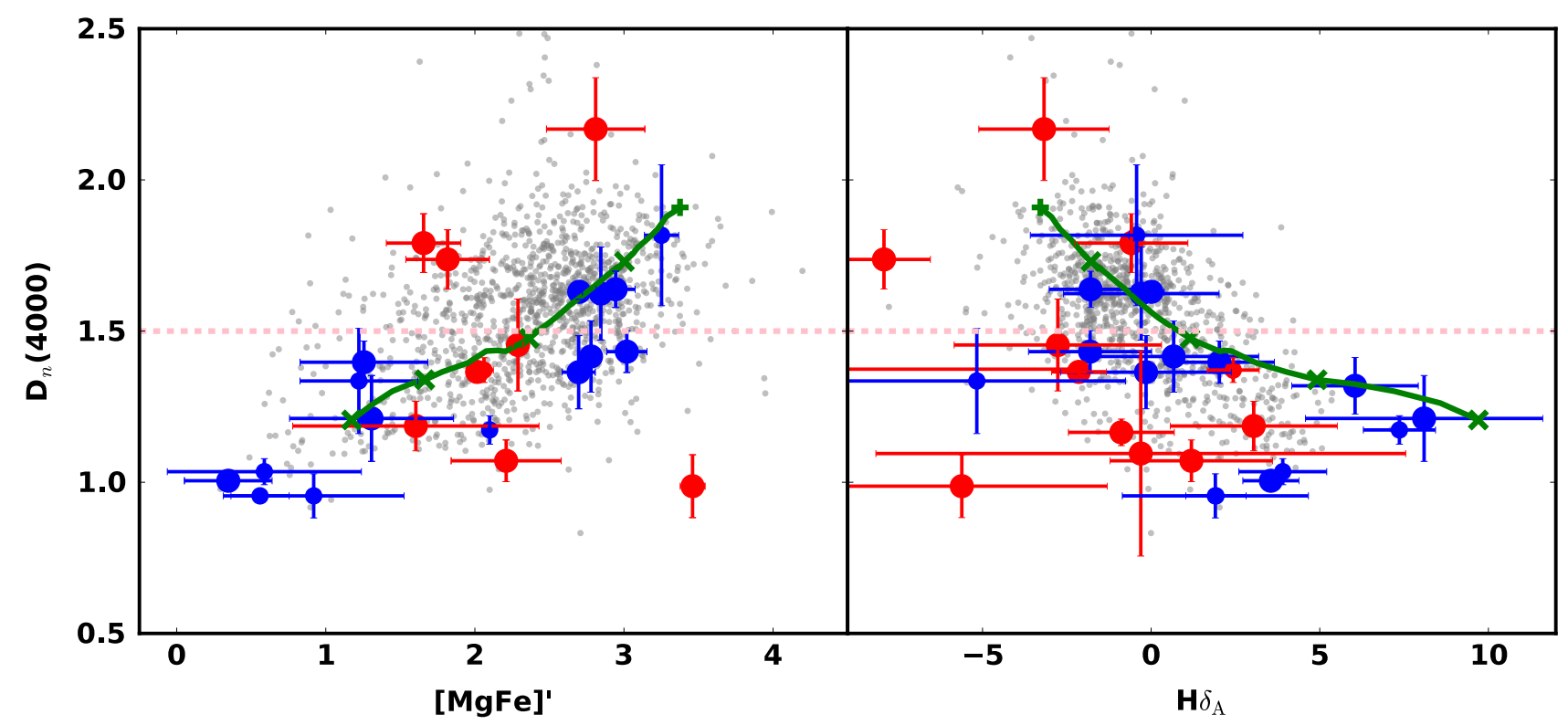

Fig. 11. Distribution of age- and metallicity-sensitive line strengths of our MUG sample. The error bars are shown at the $1 \sigma$ level. The blue (red) circles show the results for galaxies with a Sérsic index $n<2.5(>2.5)$, with their sizes referring to their stellar masses $\left(6-8 \times 10^{10} M_{\odot}\right.$ are depicted by small circles while $>8 \times 10^{10} M_{\odot}$ are the big circles). For reference, we include as grey dots the measurements of the general sample of GAMA massive galaxies $\left(>10^{11} M_{\odot}\right)$ with AAT/AAOmega spectra. The horizontal dashed line - at $D_{n}(4000)=1.5-$ splits the sample between old (above the line) and young populations. The green lines trace the evolution of a simple stellar population at solar metallicity (see text for details).

experienced galaxy mergers - due to their inherent large relative velocities - and thus maintain their compact sizes across cosmic time. A confirmation of the above scenario is only possible by means of a high-completeness, large-area spectroscopic survey, such as GAMA. However, given the extremely low number density of these objects, a wider coverage - at similar levels of completeness - would be required to unambiguously determine the channels that lead to massive compact galaxies at low redshift. Nevertheless, we can state beyond doubt (Sect. 3.5, Fig. 13) that a sizeable number of our massive compact galaxies do not reside in overdense environments, at odds with theoretical predictions.

Our sample shows no trend with group mass. Looking at dark matter halo mergers only, Fakhouri \& Ma (2010) have shown that the average number of mergers a halo encounters is both a strong function of the amount of elapsed time and the final mass of the halo. More massive groups should have undergone more mergers on average. In light of this, our results show an opposite trend if we identify MUGs as proxies for a lack of merger activity in the past. As MUGs are almost equally distributed among groups of different masses (again see Sect. 3.5, Fig. 13), the relative fraction of MUGs is higher in massive groups, since they are exponentially less abundant than low mass groups. This implies two possible scenarios, (1) either the sample of MUGs reside in host halos that underwent an atypical growth history, or (2) dynamical effects associated with galaxy mergers play an important role. It is to be expected that both of these will play a role. Theoretical models following the merger of galaxies within merging halos using dynamical friction estimates have shown that the size evolution of massive early-type galaxies with $M_{\star}>5 \times 10^{10} M_{\odot}$ are to first order independent of the halo/group mass for massive galaxies (e.g. Khochfar \& Silk 2006), which would support that part of the observed trend could be driven by the merger process of galaxies once their halos have merged. It is well established that once the velocity dispersion of galaxies in massive clusters becomes too high, merging will effectively cease to happen.

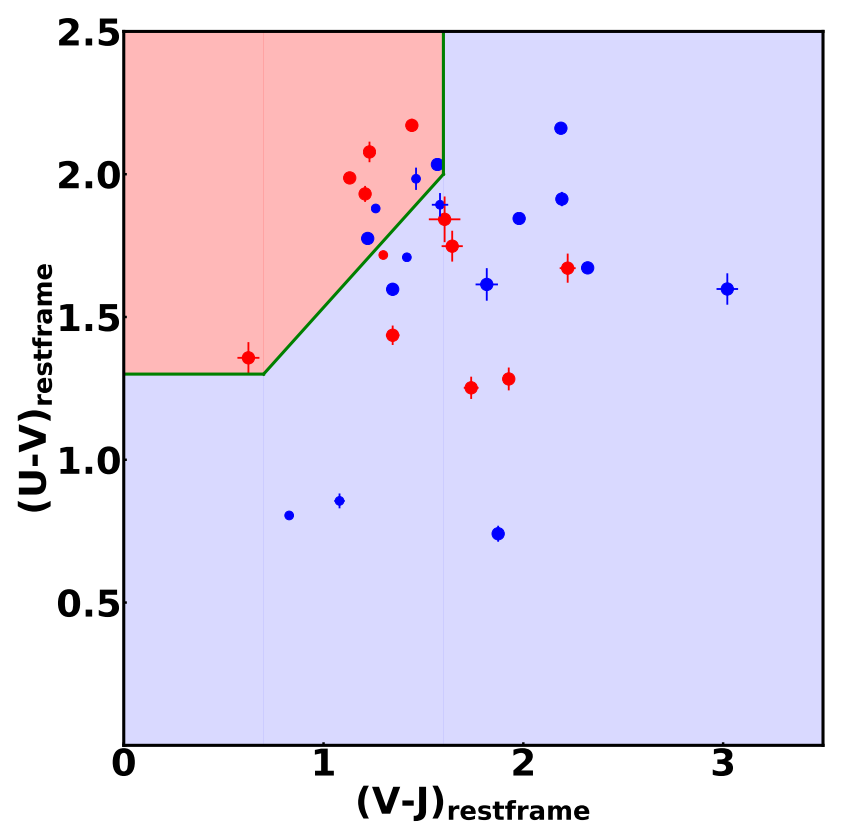

Fig. 12. Rest-frame UVJ diagram (as defined in Williams et al. 2009) for our MUG sample. The color and size coding is the same as for the rest of the figures: blue and red points distinguish disk-like and spheroid like objects ( $n<2.5$ and $n>2.5$, respectively), while small and big points denote objects with small and large stellar masses $\left(6-8 \times 10^{10}\right.$ and $>8 \times 10^{10} M_{\odot}$ ). The red and blue coloured regions separate quiescent from star-forming galaxies.

The above considerations lead to the following scenarios for the origin of MUGs: a) the passive sub-sample of early-types most-likely formed in halos that showed a fast growth history at high redshift and assembled enough mass quickly to increase the velocity dispersion of galaxies within the halo hindering mergers, especially minor mergers; b) the population of compact 


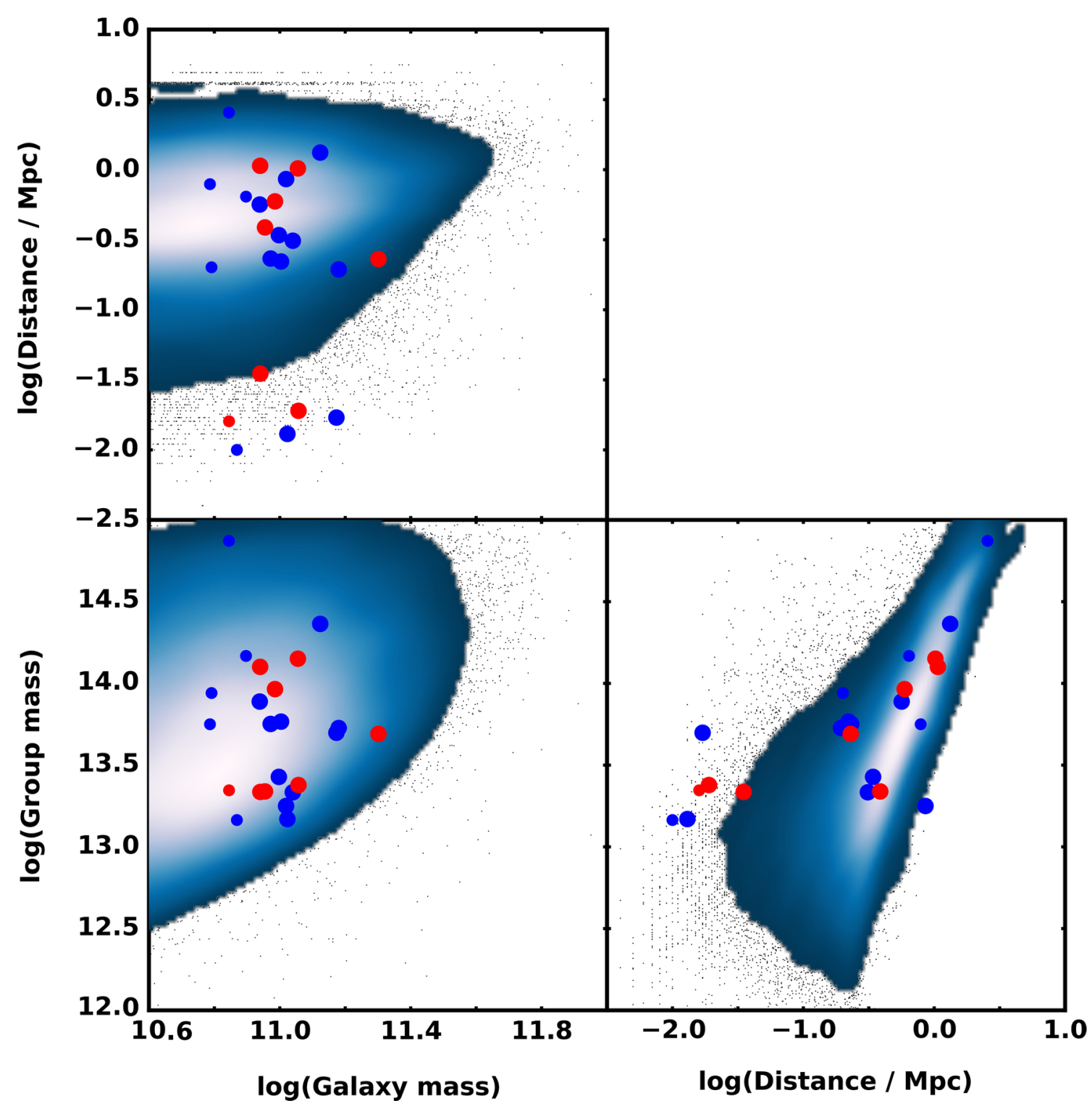

Fig. 13. Environment of our MUG sample is compared with the general distribution of GAMA massive galaxies. This is depicted by a blue colored Kernel Density Estimation, using individual datapoints where the density of datapoints is too low. Our MUG sample is colour and size coded as in Fig. 11, split according to the Sérsic index into disk-like $(n<2.5$, blue) and spheroid-like $(n>2.5$, red). The group dynamical masses and cluster-centric distances distances are taken from the $\mathrm{G}^{3} \mathrm{C}$ catalogue of Robotham et al. (2011), see text for details.

disc-like systems with ongoing star formation, in contrast, resides in halos that assembled at later times and, thus, merging of galaxies did not have enough time to proceed, or the environment may prevent mergers, depending on the group mass.

Regarding the comoving number densities of MUGs, the combination of our results with previous work from the literature suggest that, at $0<z<0.3$, these objects have number densities $\sim 10^{-6} \mathrm{Mpc}^{-3}$ (see Table 3 and Fig. 14). This value indicates that this galaxy population is very rare, and also imposes an upper limit on the density of relic galaxies, which, by definition, (massive and small vs massive and small and old) must be even less numerous. Remarkably, the MUG/“red nugget" number density is $\sim 10^{-4} \mathrm{Mpc}^{-3}$ at $z=2-3$, implying that less than one in a hundred of them survive untouched from their formation epoch. When comparing these results with simulations on a density vs redshift diagram (see Fig. 14), the data points lay in a region where the galaxies increased their stellar mass by less than $10 \%$ across cosmic time. This result illus- trates the uniqueness of these galaxies, suggesting a minimal contribution from merging events. Upcoming surveys such as WAVES (Driver et al. 2016), covering wider regions of the sky, and deeper in flux than GAMA, will allow us to produce a high enough number of MUGs, to explore their formation channels in detail.

It is remarkable that our sample of massive compact galaxies appear - at least in projection - in a relatively crowded environment (see Figs 1 and 2), complicating the photometric and spectroscopic analysis. Therefore, the inferred sizes and densities only provide upper bounds, as the light from the minor companions may contaminate the surface brightness profiles in a way that is impossible to mitigate at the spatial resolutions available to us. Follow-up observations, including imaging at high spatial resolution with HST or JWST, and deeper spectroscopy with IFU cameras at 8-10m-class telescopes, such as MUSE or MEGARA, will be required to better comprehend the intriguing properties of massive ultracompact galaxies. 
Table 3. Comoving number density of MUGs.

\begin{tabular}{ccccccc}
\hline \hline & $\begin{array}{c}\text { All galaxies } \\
\text { \#Objects }\end{array}$ & $\begin{array}{c}\text { Num. density } \\
\times 10^{-7} \mathrm{Mpc}^{-3}\end{array}$ & $\begin{array}{c}M_{10}>8 \\
\text { \#Objects }\end{array}$ & $\begin{array}{c}\text { Num. density } \\
\times 10^{-7} \mathrm{Mpc}^{-3}\end{array}$ & $\begin{array}{c}6<M_{10}<8 \\
\text { \#Objects }\end{array}$ & $\begin{array}{c}\text { Num. density } \\
\times 10^{-7} \mathrm{Mpc}^{-3}\end{array}$ \\
\hline $0.02<z<0.1$ & 0 & $<7.53$ & - & - & - & - \\
$0.1<z<0.2$ & 8 & $9.29_{-3.11}^{+4.58}$ & 7 & $8.12_{-3.00}^{+4.38}$ & 1 & $1.16_{-0.96}^{+2.67}$ \\
$0.2<z<0.3$ & 21 & $9.92_{-2.15}^{+2.67}$ & 15 & $7.08_{-1.81}^{+2.34}$ & 6 & $2.83_{-1.12}^{+1.69}$ \\
\hline
\end{tabular}

Notes. $M_{10}$ is defined as the stellar mass of the galaxy in units of $10^{10} M_{\odot}$. Error bars stem from the calculations in Gehrels (1986) for low number statistics.

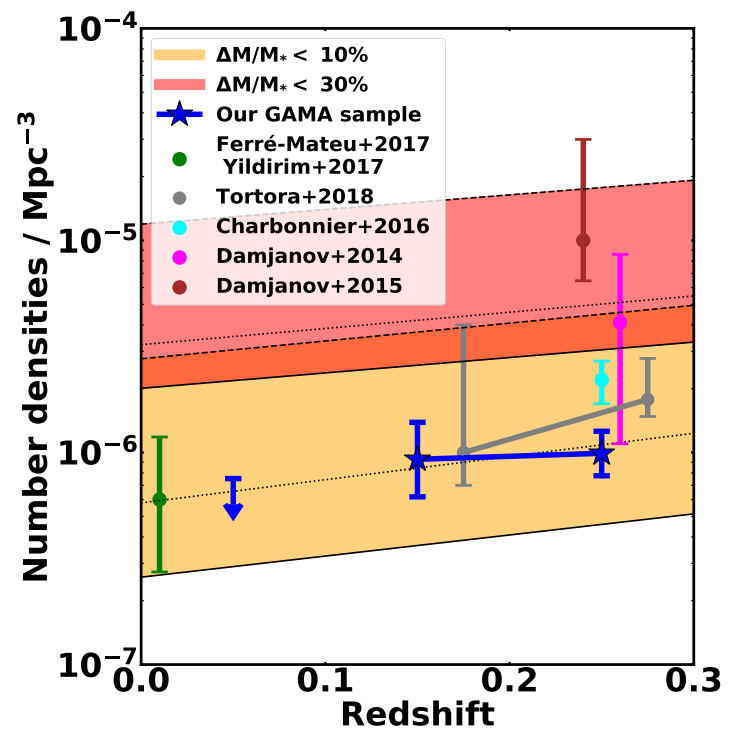

Fig. 14. Redshift evolution of the MUG comoving number density over the redshift range $0.02<z<0.3$, along with the theoretical expectations (shaded regions) taken from Fig. 2 in Quilis \& Trujillo (2013). See Sect. 3.6 for details.

Acknowledgements. FB acknowledges the support by FCT via the postdoctoral fellowship SFRH/BPD/103958/2014. This work is supported by Fundação para a Ciência e a Tecnologia (FCT) through national funds (UID/FIS/04434/2013) and by FEDER through COMPETE2020 (POCI-01-0145-FEDER-007672). FB also acknowledges support from grant AYA2016-77237-C3-1-P from the Spanish Ministry of Economy and Competitiveness (MINECO). We warmly thank the anonymous referee for a very constructive report, that has helped to clarify the manuscript's text. We also want to acknowledge K. Kuijken and M. Viola for providing us with the shapelet PSFs for our photometric analysis, and I. Trujillo and V. Quilis kindly provided us with the data of Fig. 2 from Quilis \& Trujillo (2013) as well. We also acknowledge the help of A. Molino, I. Matute, S. Reis and P. Norberg. This research made use of APLpy, an open-source plotting package for Python (Robitaille \& Bressert 2015). We have used extensively the following software packages: TOPCAT (Taylor 2005), ALADIN (Bonnarel et al. 2000) and MATPLOTLIB (Hunter 2007). GAMA is a joint European-Australasian project based around a spectroscopic campaign using the AAT. The GAMA input catalogue is based on data taken from the SDSS and the UKIRT Infrared Deep Sky Survey. Complementary imaging of the GAMA regions is being obtained by a number of independent survey programmes including GALEX MIS, VST KIDS, VISTA VIKING, WISE, Herschel-ATLAS, GMRT and ASKAP providing UV to radio coverage. GAMA is funded by the STFC (UK), the ARC (Australia), the AAO and the participating institutions. The GAMA web site is http://www. gama-survey.org/

\section{References}

Abazajian, K. N., Adelman-McCarthy, J. K., Agüeros, M. A., et al. 2009, ApJS, 182,543

Anderson, T. W., \& Darling, D. A. 1952, Ann. Math. Statist., 23, 193

Baldry, I. K., Robotham, A. S. G., Hill, D. T., et al. 2010, MNRAS, 404, 86

Baldry, I. K., Driver, S. P., Loveday, J., et al. 2012, MNRAS, 421, 621
Baldry, I. K., Liske, J., Brown, M. J. I., et al. 2018, MNRAS, 474, 3875 Balogh, M. L., Morris, S. L., Yee, H. K. C., Carlberg, R. G., \& Ellingson, E. 1999, ApJ, 527, 54

Barro, G., Faber, S. M., Pérez-González, P. G., et al. 2013, ApJ, 765, 104 Beasley, M. A., Trujillo, I., Leaman, R., \& Montes, M. 2018, Nature, 555, 483 Bertin, E., \& Arnouts, S. 1996, A\&AS, 117, 393

Bezanson, R., van Dokkum, P. G., Tal, T., et al. 2009, ApJ, 697, 1290

Bonnarel, F., Fernique, P., Bienaymé, O., et al. 2000, A\&AS, 143, 33

Brough, S., Croom, S., Sharp, R., et al. 2013, MNRAS, 435, 2903

Bruce, V. A., Dunlop, J. S., Cirasuolo, M., et al. 2012, MNRAS, 427, 1666

Bruzual, G., \& Charlot, S. 2003, MNRAS, 344, 1000

Buitrago, F., Trujillo, I., Conselice, C. J., et al. 2008, ApJ, 687, L61

Buitrago, F., Trujillo, I., Conselice, C. J., \& Häußler, B. 2013, MNRAS, 428, 1460

Buitrago, F., Conselice, C. J., Epinat, B., et al. 2014, MNRAS, 439, 1494 Buitrago, F., Trujillo, I., Curtis-Lake, E., et al. 2017, MNRAS, 466, 4888 Cardelli, J. A., Clayton, G. C., \& Mathis, J. S. 1989, ApJ, 345, 245

Carrasco, E. R., Conselice, C. J., \& Trujillo, I. 2010, MNRAS, 405, 2253

Cassata, P., Giavalisco, M., Guo, Y., et al. 2010, ApJ, 714, L79

Cassata, P., Giavalisco, M., Guo, Y., et al. 2011, ApJ, 743, 96

Chabrier, G. 2003, PASP, 115, 763

Charbonnier, A., Huertas-Company, M., Gonçalves, T. S., et al. 2017, MNRAS, 469,4523

Cimatti, A., Cassata, P., Pozzetti, L., et al. 2008, A\&A, 482, 21

Colless, M., Dalton, G., Maddox, S., et al. 2001, MNRAS, 328, 1039

Conselice, C. J., Bluck, A. F. L., Buitrago, F., et al. 2011, MNRAS, 413, 80

Daddi, E., Renzini, A., Pirzkal, N., et al. 2005, ApJ, 626, 680

Damjanov, I., McCarthy, P. J., Abraham, R. G., et al. 2009, ApJ, 695, 101

Damjanov, I., Hwang, H. S., Geller, M. J., \& Chilingarian, I. 2014, ApJ, 793, 39

Damjanov, I., Zahid, H. J., Geller, M. J., \& Hwang, H. S. 2015, ApJ, 815, 104

de Jong, J. T. A., Verdois Kleijn, G. A., Erben, T., et al. 2017, A\&A, 604, A134

de la Rosa, I. G., La Barbera, F., Ferreras, I., et al. 2016, MNRAS, 457, 1916

De Lucia, G., \& Blaizot, J. 2007, MNRAS, 375, 2

Díaz-García, L. A., Cenarro, A. J., López-Sanjuan, C., et al. 2017, A\&A, submitted, [arXiv:1711.10590]

Driver, S. P., Hill, D. T., Kelvin, L. S., et al. 2011, MNRAS, 413, 971

Driver, S. P., Robotham, A. S. G., Bland-Hawthorn, J., et al. 2013, MNRAS, 430, 2622

Driver, S. P., Davies, L. J., Meyer, M., et al. 2016, The Universe of Digital Sky Surveys, 42, 205

Edge, A., Sutherland, W., Kuijken, K., et al. 2013, The Messenger, 154, 32 Emsellem, E. 2013, MNRAS, 433, 1862

Fabian, A. C., Sanders, J. S., Haehnelt, M., Rees, M. J., \& Miller, J. M. 2013, MNRAS, 431, L38

Fakhouri, O., \& Ma, C.-P. 2010, MNRAS, 401, 2245

Ferré-Mateu, A., Vazdekis, A., \& de la Rosa, I. G. 2013, MNRAS, 431, 440

Ferré-Mateu, A., Mezcua, M., Trujillo, I., Balcells, M., \& van den Bosch, R. C. E. 2015, ApJ, 808, 79

Ferré-Mateu, A., Trujillo, I., Martín-Navarro, I., et al. 2017, MNRAS, 467, 1929

Ferreras, I., Pasquali, A., Khochfar, S., et al. 2012, AJ, 144, 47

Gehrels, N. 1986, ApJ, 303, 336

Genel, S., Nelson, D., Pillepich, A., et al. 2018, MNRAS, 474, 3976

Graham, A. W., Dullo, B. T., \& Savorgnan, G. A. D. 2015, ApJ, 804, 32

Graham, A. W., Durré, M., Savorgnan, G. A. D., et al. 2016, ApJ, 819, 43

Guo, Q., White, S., Boylan-Kolchin, M., et al. 2011, MNRAS, 413, 101

Guo, Q., White, S., Angulo, R. E., et al. 2013, MNRAS, 428, 1351

Hopkins, P. F., Bundy, K., Murray, N., et al. 2009, MNRAS, 398, 898

Hunter, J. D. 2007, Comput. Sci. Eng., 9, 90

Johansson, P. H., Naab, T., \& Ostriker, J. P. 2012, ApJ, 754, 115

Kelvin, L. S., Driver, S. P., Robotham, A. S. G., et al. 2012, MNRAS, 421, 1007

Kelvin, L. S., Driver, S. P., Robotham, A. S. G., et al. 2014, MNRAS, 444, 1647

Khochfar, S., \& Silk, J. 2006, ApJ, 648, L21 
Kuijken, K., Heymans, C., Hildebrandt, H., et al. 2015, MNRAS, 454, 3500 Lange, R., Driver, S. P., Robotham, A. S. G., et al. 2015, MNRAS, 447, 2603 Lapi, A., Pantoni, L., Zanisi, L., et al. 2018, ApJ, 857, 22

Liske, J., Baldry, I. K., Driver, S. P., et al. 2015, MNRAS, 452, 2087

Magorrian, J., Tremaine, S., Richstone, D., et al. 1998, AJ, 115, 2285

Martín-Navarro, I., Pérez-González, P. G., Trujillo, I., et al. 2015, ApJ, 798, L4

McLure, R. J., Pearce, H. J., Dunlop, J. S., et al. 2013, MNRAS, 428, 1088

Naab, T., Johansson, P. H., \& Ostriker, J. P. 2009, ApJ, 699, L178

Nipoti, C., Treu, T., Leauthaud, A., et al. 2012, MNRAS, 422, 1714

Oke, J. B., \& Gunn, J. E. 1983, ApJ, 266, 713

Oser, L., Ostriker, J. P., Naab, T., Johansson, P. H., \& Burkert, A. 2010, ApJ, 725 2312

Peng, C. Y., Ho, L. C., Impey, C. D., \& Rix, H.-W. 2002, AJ, 124, 266

Peng, C. Y., Ho, L. C., Impey, C. D., \& Rix, H.-W. 2010, AJ, 139, 2097

Peralta de Arriba, L., Quilis, V., Trujillo, I., Cebrián, M., \& Balcells, M. 2016 MNRAS, 461, 156

Poggianti, B. M., Calvi, R., Bindoni, D., et al. 2013, ApJ, 762, 77

Quilis, V., \& Trujillo, I. 2013, ApJ, 773, L8

Robitaille, T., \& Bressert, E. 2012, Astrophysics Source Code Library [record ascl:1208.017]

Robotham, A., Driver, S. P., Norberg, P., et al. 2010, PASA, 27, 76

Robotham, A. S. G., Norberg, P., Driver, S. P., et al. 2011, MNRAS, 416, 2640

Saulder, C., van den Bosch, R. C. E., \& Mieske, S. 2015, A\&A, 578, A134

Scharwächter, J., Combes, F., Salomé, P., Sun, M., \& Krips, M. 2016, MNRAS, 457,4272

Schlafly, E. F., \& Finkbeiner, D. P. 2011, ApJ, 737, 103

Sérsic, J. L. 1968, Atlas de Galaxias Australes, ed. J. L. Sérsic (Córdoba, Argentina: Observatorio Astronómico)

Shen, S., Mo, H. J., White, S. D. M., et al. 2003, MNRAS, 343, 978

Shih, H.-Y., \& Stockton, A. 2011, ApJ, 733, 45

Stockton, A., Shih, H.-Y., Larson, K., \& Mann, A. W. 2014, ApJ, 780, 134

Stringer, M., Trujillo, I., Dalla Vecchia, C., \& Martinez-Valpuesta, I. 2015, MNRAS, 449, 2396

Strom, S. E., \& Strom, K. M. 1978, ApJ, 225, L93

Taylor, E. N., Franx, M., Glazebrook, K., et al. 2010, ApJ, 720, 723
Taylor, E. N., Hopkins, A. M., Baldry, I. K., et al. 2011, MNRAS, 418, 1587

Taylor, M. B. 2005, in Astronomical Data Analysis Software and Systems XIV, eds. P. Shopbell, M. Britton, \& R. Ebert, ASP Conf. Ser., 347, 29

Thomas, D., Maraston, C., \& Bender, R. 2003, MNRAS, 339, 897

Tonry, J. L., Blakeslee, J. P., Ajhar, E. A., \& Dressler, A. 2000, ApJ, 530, 625

Tortora, C., La Barbera, F., Napolitano, N. R., et al. 2016, MNRAS, 457, 2845

Tortora, C., Napolitano, N. R., Spavone, M., et al. 2018, MNRAS, 481, 4728

Trujillo, I., Feulner, G., Goranova, Y., et al. 2006, MNRAS, 373, L36

Trujillo, I., Conselice, C. J., Bundy, K., et al. 2007, MNRAS, 382, 109

Trujillo, I., Cenarro, A. J., de Lorenzo-Cáceres, A., et al. 2009, ApJ, 692, L118

Trujillo, I., Ferreras, I., \& de La Rosa, I. G. 2011, MNRAS, 415, 3903

Trujillo, I., Carrasco, E. R., \& Ferré-Mateu, A. 2012, ApJ, 751, 45

Trujillo, I., Ferré-Mateu, A., Balcells, M., Vazdekis, A., \& Sánchez-Blázquez, P. 2014, ApJ, 780, L20

Valentinuzzi, T., Fritz, J., Poggianti, B. M., et al. 2010a, ApJ, 712, 226

Valentinuzzi, T., Poggianti, B. M., Saglia, R. P., et al. 2010b, ApJ, 721, L19

van den Bosch, R. C. E., Gebhardt, K., Gültekin, K., et al. 2012, Nature, 491, 729

van der Wel, A., Rix, H.-W., Wuyts, S., et al. 2011, ApJ, 730, 38

van der Wel, A., Franx, M., van Dokkum, P. G., et al. 2014, ApJ, 788, 28

van Dokkum, P. G., Whitaker, K. E., Brammer, G., et al. 2010, ApJ, 709, 1018

Vazdekis, A., Ricciardelli, E., Cenarro, A. J., et al. 2012, MNRAS, 424, 157

Viola, M., Cacciato, M., Brouwer, M., et al. 2015, MNRAS, 452, 3529

Wellons, S., Torrey, P., Ma, C.-P., et al. 2016, MNRAS, 456, 1030

Williams, R. J., Quadri, R. F., Franx, M., van Dokkum, P., \& Labbé, I. 2009. ApJ, 691, 1879

Worthey, G., \& Ottaviani, D. L. 1997, ApJS, 111, 377

Yıldırım, A., van den Bosch, R. C. E., van de Ven, G., et al. 2015, MNRAS, 452, 1792

Yıldırım, A., van den Bosch, R. C. E., van de Ven, G., et al. 2017, MNRAS, 468, 4216

Zolotov, A., Dekel, A., Mandelker, N., et al. 2015, MNRAS, 450, 2327

Zwicky, F., \& Kowal, C. T. 1968, Catalogue of Galaxies and of Clusters of Galaxies, VI 


\section{Appendix A: Robustness of the measured structural parameters}

We simulated 3840 galaxies, both in the KiDS $r$-band and the VIKING Z-band, with the tools described in Buitrago et al. (2013). We explored the range of structural parameters shown by our sample, namely:

$$
\begin{aligned}
& 15<\operatorname{mag}<20 \\
& 1<R_{\mathrm{e}} \mathrm{pix}^{-1}<10 \\
& 1<n<8 \\
& 0.3<\text { ar }<1 \\
& 0<p a<90
\end{aligned}
$$

where mag, $R_{\mathrm{e}}, n$, ar and $p a$ stand out for magnitude (either in the $r$ - or $Z$-band), effective radius, Sérsic index, axis ratio and position angle.
The structural parameters of the mock galaxies were randomly distributed in a linear way, considering the full parameter space defined by the previously mentioned values. The mock galaxies were placed randomly on the KiDS and VIKING images, only imposing the condition that they should not overlap with any detection defined by the SExtractor segmentation map of each survey. Each mock galaxy was convolved with a representative PSF from those images, and analyzed with the same code we utilize to investigate the real galaxies.

According to Figs. A.1 and A.2, our ability to recuperate the structural parameters degrades at fainter fluxes and higher Sérsic indices. This result is expected, because dimmer and more concentrated objects are more difficult to analyze (a similar behaviour is found in the simulations of Buitrago et al. 2013). In the $Z$-band, the results are somewhat worse because of the coarser pixel scale $\left(0.21 \operatorname{arcsec~pix}^{-1}\right.$ versus $\left.0.339 \operatorname{arcsec~pix}^{-1}\right)$. However, for the data at hand, caution needs to be taken only for faint objects with a high Sérsic index.

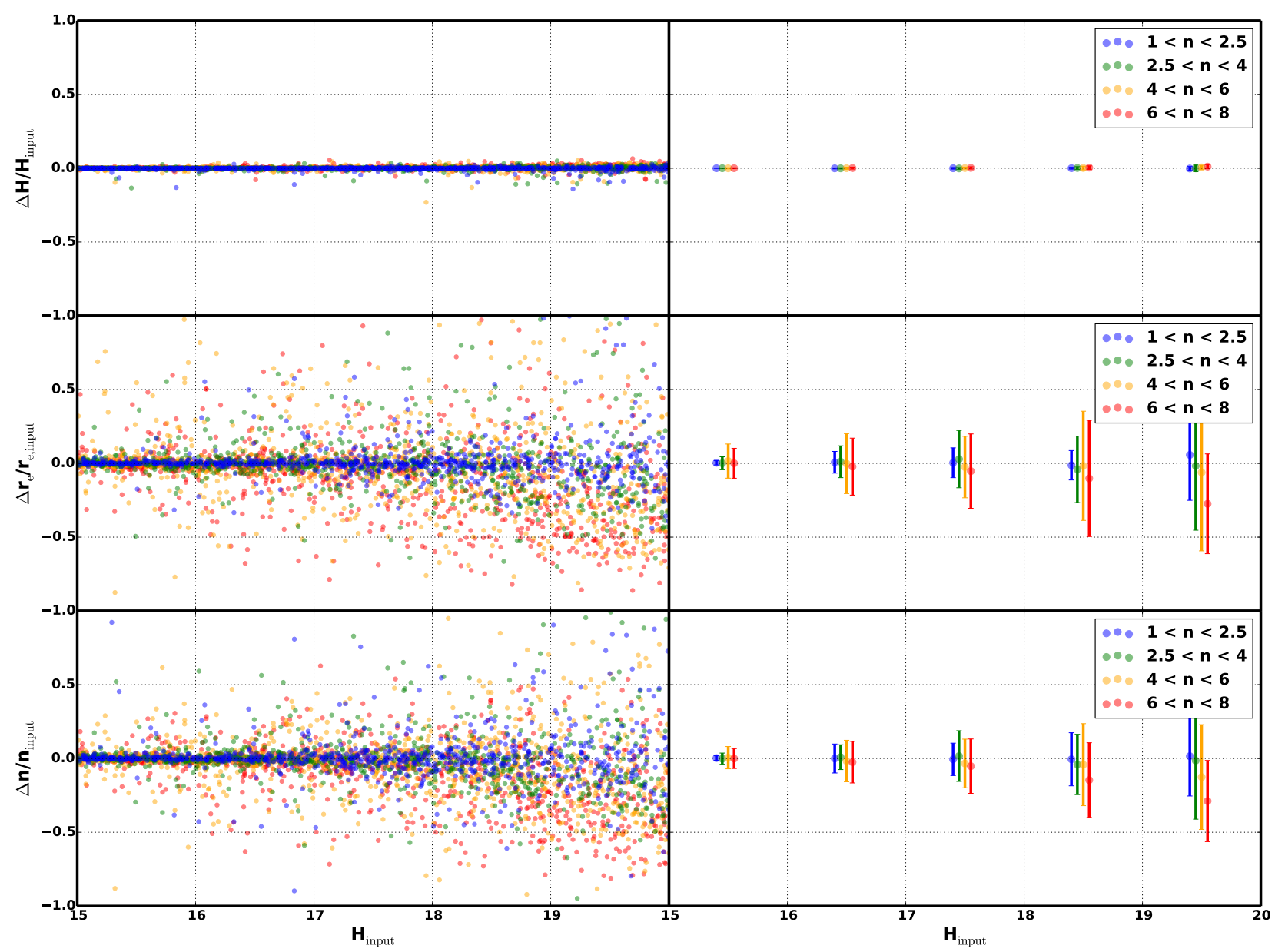

Fig. A.1. Relative errors - (output-input)/input - of the structural parameters (magnitude, effective radius and Sérsic index) of our simulated KiDS galaxies in the $r$-band. The right-hand column shows the average values in 1 mag bins (derived after a $5 \sigma$ clipping). The error bars represent the standard deviation within each bin. 


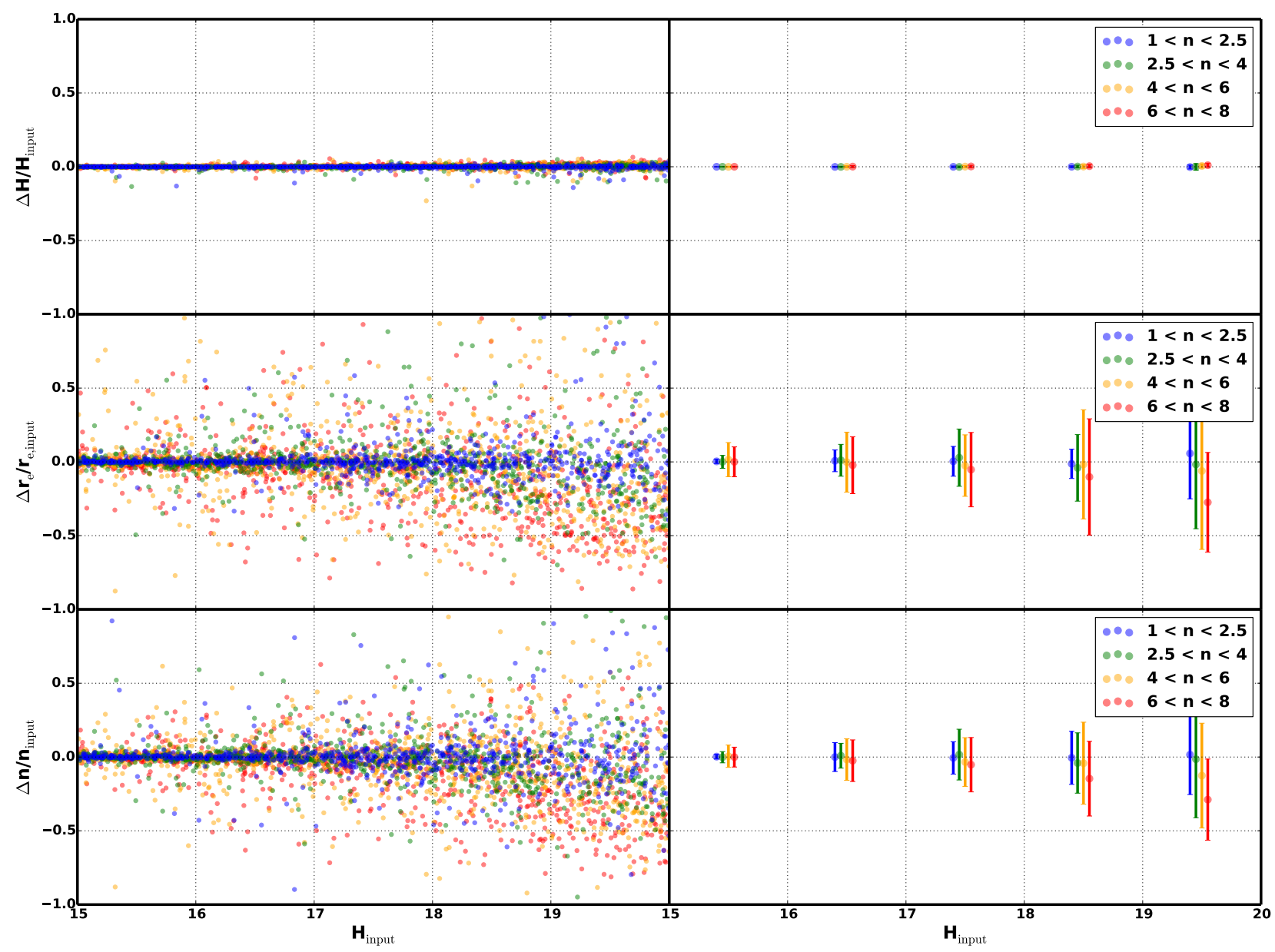

Fig. A.2. Relative errors - (output-input)/input - of the structural parameters (magnitude, effective radius and Sérsic index) of our simulated VIKING galaxies in the $Z$-band. The right-hand column shows the average values in 1 mag bins (derived after a $5 \sigma$ clipping). The error bars represent the standard deviation within each bin. 
F. Buitrago et al.: Number densities and environments for low-z massive ultra-compact galaxies

\section{Appendix B: Structural parameters for the $g$-, $i$ - and Z-bands}

Table B.1. $g$-band structural parameters for our MUG sample.

\begin{tabular}{|c|c|c|c|c|c|}
\hline CATAID & $\operatorname{mag} \pm \delta \mathrm{mag}$ & $\begin{array}{c}r_{\mathrm{e}} \pm \delta r_{\mathrm{e}} \\
(\mathrm{kpc})\end{array}$ & $\begin{array}{c}r_{\mathrm{e}, \mathrm{circ}} \pm \delta r_{\mathrm{e}, \mathrm{circ}} \\
(\mathrm{kpc})\end{array}$ & $n \pm \delta n$ & $a r \pm \delta a r$ \\
\hline 79071 & $17.83 \pm 0.01$ & $2.37 \pm 0.01$ & $1.98 \pm 0.14$ & $2.13 \pm 0.05$ & $0.70 \pm 0.10$ \\
\hline 16143 & $19.65 \pm 0.01$ & $1.54 \pm 0.06$ & $1.34 \pm 0.18$ & $3.87 \pm 0.19$ & $0.76 \pm 0.15$ \\
\hline 784327 & $20.51 \pm 0.12$ & $1.21 \pm 0.22$ & $1.11 \pm 0.22$ & $2.21 \pm 0.44$ & $0.85 \pm 0.03$ \\
\hline 609701 & $20.79 \pm 0.09$ & $1.54 \pm 0.46$ & $1.51 \pm 0.57$ & $1.62 \pm 0.75$ & $0.97 \pm 0.14$ \\
\hline 493286 & $21.22 \pm 0.09$ & $1.34 \pm 0.07$ & $1.26 \pm 0.29$ & $1.75 \pm 0.62$ & $0.87 \pm 0.30$ \\
\hline 319149 & $20.38 \pm 0.05$ & $2.57 \pm 0.22$ & $2.20 \pm 0.43$ & $2.62 \pm 0.46$ & $0.73 \pm 0.16$ \\
\hline 84466 & $20.69 \pm 0.19$ & $1.43 \pm 0.24$ & $1.22 \pm 0.37$ & $1.42 \pm 0.82$ & $0.72 \pm 0.20$ \\
\hline 178241 & $20.25 \pm 0.40$ & $2.58 \pm 0.46$ & $2.44 \pm 0.49$ & $3.03 \pm 1.13$ & $0.90 \pm 0.03$ \\
\hline 93202 & $18.60 \pm 0.01$ & $2.27 \pm 0.01$ & $2.12 \pm 0.12$ & $1.80 \pm 0.09$ & $0.88 \pm 0.10$ \\
\hline 63726 & $21.10 \pm 0.05$ & $3.24 \pm 0.19$ & $2.48 \pm 0.85$ & $7.01 \pm 0.45$ & $0.59 \pm 0.33$ \\
\hline 765033 & $20.92 \pm 0.01$ & $3.09 \pm 0.01$ & $2.05 \pm 0.22$ & $0.32 \pm 0.13$ & $0.44 \pm 0.09$ \\
\hline 4220443 & $21.34 \pm 0.01$ & $1.64 \pm 0.27$ & $1.21 \pm 0.35$ & $2.31 \pm 1.10$ & $0.55 \pm 0.13$ \\
\hline 422365 & $21.36 \pm 0.10$ & $1.72 \pm 0.53$ & $1.72 \pm 0.65$ & $3.41 \pm 0.63$ & $1.00 \pm 0.15$ \\
\hline 71471 & $20.59 \pm 0.09$ & $1.70 \pm 0.08$ & $1.42 \pm 0.16$ & $5.44 \pm 0.33$ & $0.71 \pm 0.09$ \\
\hline 3873542 & $20.20 \pm 0.02$ & $0.87 \pm 0.06$ & $0.82 \pm 0.16$ & $2.77 \pm 0.08$ & $0.89 \pm 0.24$ \\
\hline 300986 & $20.83 \pm 0.09$ & $0.69 \pm 0.04$ & $0.57 \pm 0.09$ & $2.39 \pm 0.59$ & $0.67 \pm 0.13$ \\
\hline 221269 & $20.52 \pm 0.20$ & $1.76 \pm 0.30$ & $1.33 \pm 0.53$ & $9.69 \pm 0.59$ & $0.57 \pm 0.27$ \\
\hline 288762 & $21.42 \pm 0.09$ & $2.71 \pm 0.11$ & $2.58 \pm 0.30$ & $1.13 \pm 0.58$ & $0.90 \pm 0.14$ \\
\hline 138954 & $20.64 \pm 0.03$ & - & - & - & - \\
\hline 855304 & $22.23 \pm 0.09$ & $0.94 \pm 0.05$ & $0.61 \pm 0.08$ & $6.51 \pm 0.50$ & $0.41 \pm 0.07$ \\
\hline 791716 & $21.54 \pm 0.01$ & $0.99 \pm 0.01$ & $0.32 \pm 0.36$ & $1.58 \pm 0.01$ & $0.11 \pm 0.23$ \\
\hline 693193 & $19.32 \pm 0.01$ & $3.67 \pm 0.03$ & $2.76 \pm 0.85$ & $4.17 \pm 0.05$ & $0.57 \pm 0.34$ \\
\hline 723783 & $19.56 \pm 0.08$ & $1.76 \pm 0.13$ & $1.49 \pm 0.33$ & $1.16 \pm 0.11$ & $0.72 \pm 0.21$ \\
\hline 486049 & $21.02 \pm 0.20$ & $2.51 \pm 0.31$ & $2.11 \pm 0.58$ & $1.58 \pm 0.71$ & $0.71 \pm 0.22$ \\
\hline 388238 & $19.96 \pm 0.09$ & $1.30 \pm 0.23$ & $1.08 \pm 0.38$ & $2.12 \pm 0.23$ & $0.70 \pm 0.24$ \\
\hline 55006 & $20.71 \pm 0.01$ & $1.72 \pm 0.37$ & $1.60 \pm 0.61$ & $3.40 \pm 1.02$ & $0.86 \pm 0.29$ \\
\hline 750374 & $21.21 \pm 0.09$ & $0.50 \pm 0.09$ & $0.33 \pm 0.12$ & $2.03 \pm 0.46$ & $0.43 \pm 0.17$ \\
\hline 3173601 & $20.41 \pm 0.09$ & - & - & - & - \\
\hline 365375 & $20.69 \pm 0.09$ & $1.56 \pm 0.11$ & $1.34 \pm 0.01$ & $1.05 \pm 0.33$ & $0.74 \pm 0.01$ \\
\hline
\end{tabular}

Notes. The division line splits the galaxies with stellar mass greater or lower than $8 \times 10^{10} M_{\odot}$. Columns: (1) GAMA ID, (2) magnitude, (3) effective radius in kpc, (4) circularized effective radius in kpc, (5) Sérsic index, (6) axis ratio. 
Table B.2. $i$-band structural parameters for our MUG sample.

\begin{tabular}{|c|c|c|c|c|c|}
\hline CATAID & $\mathrm{mag} \pm \delta \mathrm{mag}$ & $\begin{array}{c}r_{\mathrm{e}} \pm \delta r_{\mathrm{e}} \\
(\mathrm{kpc})\end{array}$ & $\begin{array}{c}r_{\mathrm{e}, \mathrm{circ}} \pm \delta r_{\mathrm{e}, \mathrm{circ}} \\
(\mathrm{kpc})\end{array}$ & $n \pm \delta n$ & $a r \pm \delta a r$ \\
\hline 79071 & $16.50 \pm 0.01$ & $2.14 \pm 0.01$ & $1.78 \pm 0.13$ & $2.01 \pm 0.05$ & $0.69 \pm 0.10$ \\
\hline 16143 & $18.00 \pm 0.01$ & $1.67 \pm 0.06$ & $1.57 \pm 0.19$ & $5.87 \pm 0.19$ & $0.88 \pm 0.15$ \\
\hline 784327 & $19.08 \pm 0.12$ & $1.18 \pm 0.22$ & $1.11 \pm 0.22$ & $1.91 \pm 0.44$ & $0.89 \pm 0.03$ \\
\hline 609701 & $19.22 \pm 0.09$ & $2.01 \pm 0.46$ & $1.93 \pm 0.60$ & $0.95 \pm 0.75$ & $0.92 \pm 0.14$ \\
\hline 493286 & $19.51 \pm 0.09$ & $1.68 \pm 0.07$ & $1.60 \pm 0.34$ & $0.95 \pm 0.62$ & $0.91 \pm 0.30$ \\
\hline 319149 & $18.77 \pm 0.05$ & $1.78 \pm 0.22$ & $1.63 \pm 0.36$ & $2.03 \pm 0.46$ & $0.83 \pm 0.16$ \\
\hline 84466 & $18.74 \pm 0.19$ & $1.99 \pm 0.24$ & $1.87 \pm 0.44$ & $1.08 \pm 0.82$ & $0.89 \pm 0.20$ \\
\hline 178241 & $18.64 \pm 0.40$ & $1.91 \pm 0.46$ & $1.68 \pm 0.45$ & $3.57 \pm 1.13$ & $0.77 \pm 0.03$ \\
\hline 93202 & $17.31 \pm 0.01$ & $2.45 \pm 0.01$ & $2.27 \pm 0.13$ & $1.39 \pm 0.09$ & $0.86 \pm 0.10$ \\
\hline 63726 & $19.44 \pm 0.05$ & $1.56 \pm 0.19$ & $1.47 \pm 0.46$ & $1.63 \pm 0.45$ & $0.88 \pm 0.33$ \\
\hline 765033 & $18.83 \pm 0.01$ & $0.68 \pm 0.01$ & $0.39 \pm 0.06$ & $5.63 \pm 0.13$ & $0.33 \pm 0.09$ \\
\hline 4220443 & $19.44 \pm 0.01$ & $1.29 \pm 0.27$ & $0.95 \pm 0.32$ & $3.52 \pm 1.10$ & $0.55 \pm 0.13$ \\
\hline 422365 & $19.10 \pm 0.10$ & $4.15 \pm 0.53$ & $3.71 \pm 0.81$ & $2.82 \pm 0.63$ & $0.80 \pm 0.15$ \\
\hline 71471 & $18.49 \pm 0.09$ & $3.09 \pm 0.08$ & $2.92 \pm 0.23$ & $1.42 \pm 0.33$ & $0.89 \pm 0.09$ \\
\hline 3873542 & $17.94 \pm 0.02$ & $1.89 \pm 0.06$ & $1.49 \pm 0.33$ & $2.86 \pm 0.08$ & $0.62 \pm 0.24$ \\
\hline 300986 & $18.60 \pm 0.09$ & $0.75 \pm 0.04$ & $0.73 \pm 0.09$ & $1.93 \pm 0.59$ & $0.95 \pm 0.13$ \\
\hline 221269 & $19.18 \pm 0.20$ & $2.13 \pm 0.30$ & $1.98 \pm 0.58$ & $0.70 \pm 0.59$ & $0.86 \pm 0.27$ \\
\hline 288762 & $19.47 \pm 0.09$ & $1.91 \pm 0.11$ & $1.34 \pm 0.26$ & $0.77 \pm 0.58$ & $0.49 \pm 0.14$ \\
\hline 138954 & $18.35 \pm 0.03$ & $1.97 \pm 0.01$ & $1.93 \pm 0.29$ & $5.12 \pm 0.54$ & $0.95 \pm 0.28$ \\
\hline 855304 & $19.36 \pm 0.09$ & $1.00 \pm 0.05$ & $0.84 \pm 0.08$ & $1.27 \pm 0.50$ & $0.71 \pm 0.07$ \\
\hline 791716 & $19.07 \pm 0.01$ & $0.81 \pm 0.01$ & $0.75 \pm 0.11$ & $1.97 \pm 0.01$ & $0.87 \pm 0.23$ \\
\hline 693193 & $17.74 \pm 0.01$ & $1.70 \pm 0.03$ & $1.11 \pm 0.46$ & $9.67 \pm 0.05$ & $0.42 \pm 0.34$ \\
\hline 723783 & $17.67 \pm 0.08$ & $0.91 \pm 0.13$ & $0.79 \pm 0.22$ & $1.16 \pm 0.11$ & $0.76 \pm 0.21$ \\
\hline 486049 & $19.22 \pm 0.20$ & $2.85 \pm 0.31$ & $2.61 \pm 0.62$ & $0.92 \pm 0.71$ & $0.84 \pm 0.22$ \\
\hline 388238 & $18.24 \pm 0.09$ & - & - & - & - \\
\hline 55006 & $19.19 \pm 0.01$ & $2.74 \pm 0.37$ & $2.51 \pm 0.76$ & $1.10 \pm 1.02$ & $0.84 \pm 0.29$ \\
\hline 750374 & $18.81 \pm 0.09$ & $0.79 \pm 0.09$ & $0.58 \pm 0.15$ & $1.71 \pm 0.46$ & $0.53 \pm 0.17$ \\
\hline 3173601 & $19.11 \pm 0.09$ & $1.05 \pm 0.06$ & $1.03 \pm 0.13$ & $1.87 \pm 0.71$ & $0.97 \pm 0.12$ \\
\hline 365375 & $19.94 \pm 0.09$ & $1.89 \pm 0.11$ & $1.83 \pm 0.01$ & $0.61 \pm 0.33$ & $0.94 \pm 0.01$ \\
\hline
\end{tabular}

Notes. The division line splits the galaxies with stellar mass greater or lower than $8 \times 10^{10} M_{\odot}$. Columns: (1) GAMA ID, (2) magnitude, (3) effective radius in kpc, (4) circularized effective radius in kpc, (5) Sérsic index, (6) axis ratio. 
Table B.3. Z-band structural parameters for our MUG sample.

\begin{tabular}{|c|c|c|c|c|c|}
\hline CATAID & $\operatorname{mag} \pm \delta \mathrm{mag}$ & $\begin{array}{l}r_{\mathrm{e}} \pm \delta r_{\mathrm{e}} \\
(\mathrm{kpc})\end{array}$ & $\begin{array}{l}r_{\mathrm{e}, \mathrm{circ}} \pm \delta r_{\mathrm{e}, \mathrm{circ}} \\
(\mathrm{kpc})\end{array}$ & $n \pm \delta n$ & $a r \pm \delta a r$ \\
\hline 79071 & $16.18 \pm 0.03$ & $1.69 \pm 0.09$ & $1.39 \pm 0.17$ & $3.57 \pm 0.24$ & $0.68 \pm 0.10$ \\
\hline 16143 & $17.75 \pm 0.02$ & $1.02 \pm 0.02$ & $0.81 \pm 0.06$ & $4.73 \pm 0.17$ & $0.63 \pm 0.07$ \\
\hline 784327 & $18.67 \pm 0.12$ & $1.33 \pm 0.09$ & $1.23 \pm 0.09$ & $1.44 \pm 0.15$ & $0.85 \pm 0.01$ \\
\hline 609701 & $18.74 \pm 0.01$ & $1.65 \pm 0.02$ & $1.45 \pm 0.08$ & $2.67 \pm 0.02$ & $0.77 \pm 0.06$ \\
\hline 493286 & $19.29 \pm 0.28$ & $1.33 \pm 0.30$ & $1.25 \pm 0.33$ & $1.21 \pm 0.51$ & $0.88 \pm 0.07$ \\
\hline 319149 & $18.33 \pm 0.04$ & $1.83 \pm 0.08$ & $1.50 \pm 0.10$ & $4.71 \pm 0.26$ & $0.68 \pm 0.03$ \\
\hline 84466 & $18.27 \pm 0.06$ & $0.91 \pm 0.05$ & $0.72 \pm 0.15$ & $3.91 \pm 0.78$ & $0.63 \pm 0.18$ \\
\hline 178241 & $18.47 \pm 0.01$ & $1.93 \pm 0.02$ & $1.79 \pm 0.05$ & $2.06 \pm 0.01$ & $0.86 \pm 0.02$ \\
\hline 93202 & $17.08 \pm 0.01$ & $1.90 \pm 0.01$ & $1.83 \pm 0.11$ & $2.60 \pm 0.23$ & $0.93 \pm 0.10$ \\
\hline 63726 & $18.76 \pm 0.03$ & $2.01 \pm 0.04$ & $1.77 \pm 0.09$ & $5.85 \pm 0.34$ & $0.78 \pm 0.05$ \\
\hline 765033 & $18.66 \pm 0.01$ & $1.24 \pm 0.02$ & $0.77 \pm 0.03$ & $2.54 \pm 0.02$ & $0.38 \pm 0.02$ \\
\hline 4220443 & $19.06 \pm 0.11$ & $1.62 \pm 0.23$ & $0.91 \pm 0.62$ & $3.89 \pm 1.56$ & $0.32 \pm 0.34$ \\
\hline 422365 & $19.13 \pm 0.11$ & $1.93 \pm 0.28$ & $1.82 \pm 0.40$ & $3.69 \pm 1.48$ & $0.89 \pm 0.14$ \\
\hline 71471 & $17.95 \pm 0.01$ & $2.37 \pm 0.01$ & $1.84 \pm 0.17$ & $5.35 \pm 0.14$ & $0.61 \pm 0.11$ \\
\hline 3873542 & $17.90 \pm 0.01$ & $1.41 \pm 0.01$ & $1.00 \pm 0.19$ & $1.88 \pm 0.06$ & $0.50 \pm 0.19$ \\
\hline 300986 & $18.14 \pm 0.06$ & $1.29 \pm 0.08$ & $1.08 \pm 0.31$ & $3.32 \pm 0.66$ & $0.70 \pm 0.32$ \\
\hline 221269 & $18.89 \pm 0.12$ & $2.76 \pm 0.18$ & $2.27 \pm 0.18$ & $0.44 \pm 0.04$ & $0.68 \pm 0.02$ \\
\hline 288762 & $19.19 \pm 0.28$ & $1.60 \pm 0.37$ & $1.26 \pm 0.31$ & $1.15 \pm 0.48$ & $0.62 \pm 0.02$ \\
\hline 138954 & $17.93 \pm 0.01$ & $0.89 \pm 0.01$ & $0.62 \pm 0.10$ & $9.59 \pm 0.02$ & $0.50 \pm 0.15$ \\
\hline 855304 & $18.14 \pm 0.01$ & $2.45 \pm 0.02$ & $1.88 \pm 0.40$ & $6.52 \pm 0.32$ & $0.59 \pm 0.25$ \\
\hline 791716 & $18.38 \pm 0.01$ & $2.12 \pm 0.03$ & $1.76 \pm 0.06$ & $2.94 \pm 0.02$ & $0.69 \pm 0.03$ \\
\hline 693193 & $17.42 \pm 0.01$ & - & - & - & - \\
\hline 723783 & $17.47 \pm 0.01$ & $0.67 \pm 0.01$ & $0.50 \pm 0.11$ & $2.59 \pm 0.23$ & $0.56 \pm 0.25$ \\
\hline 486049 & $18.92 \pm 0.12$ & $1.64 \pm 0.11$ & $1.31 \pm 0.22$ & $1.81 \pm 0.18$ & $0.64 \pm 0.13$ \\
\hline 388238 & $17.60 \pm 0.01$ & - & - & - & - \\
\hline 55006 & $18.85 \pm 0.01$ & $1.43 \pm 0.02$ & $1.03 \pm 0.11$ & $2.81 \pm 0.02$ & $0.52 \pm 0.10$ \\
\hline 750374 & $18.17 \pm 0.01$ & - & - & - & - \\
\hline 3173601 & $18.97 \pm 0.12$ & $1.15 \pm 0.08$ & $0.69 \pm 0.31$ & $0.64 \pm 0.06$ & $0.36 \pm 0.27$ \\
\hline 365375 & $18.97 \pm 0.02$ & $1.89 \pm 0.02$ & $1.22 \pm 0.01$ & $2.48 \pm 0.02$ & $0.41 \pm 0.01$ \\
\hline
\end{tabular}

Notes. The division line splits the galaxies with stellar mass greater or lower than $8 \times 10^{10} M_{\odot}$. Columns: (1) GAMA ID, (2) magnitude, (3) effective radius in kpc, (4) circularized effective radius in kpc, (5) Sérsic index, (6) axis ratio. 